Helsinki University of Technology Institute of Mathematics Research Reports

Teknillisen korkeakoulun matematiikan laitoksen tutkimusraporttisarja

Espoo 2004

\title{
A POSTERIORI ERROR ANALYSIS \\ OF THE LINKED INTERPOLATION TECHNIQUE FOR PLATE BENDING PROBLEMS
}

Carlo Lovadina Rolf Stenberg 

Helsinki University of Technology Institute of Mathematics Research Reports

Teknillisen korkeakoulun matematiikan laitoksen tutkimusraporttisarja

Espoo 2004

\section{A POSTERIORI ERROR ANALYSIS \\ OF THE LINKED INTERPOLATION TECHNIQUE FOR PLATE BENDING PROBLEMS}

Carlo Lovadina Rolf Stenberg 
Carlo Lovadina and Rolf Stenberg: A posteriori error analysis of the linked interpolation technique for plate bending problems; Helsinki University of Technology Institute of Mathematics Research Reports A472 (2004).

Abstract: We develop a posteriori error estimates for the so-called'Linked Interpolation Technique' to approximate the solution of plate bending problems. We show that the proposed (residual-based) estimator is both reliable and efficient.

AMS subject classifications: Primary 65N30; Secondary 74S05.

Keywords: Reissner-Mindlin plates, finite element methods, a posteriori error analysis.

Correspondence: Carlo Lovadina, Dipartimento di Matematica, Università di Pavia, and IMATI-CNR, Via Ferrata 1, Pavia I-27100, Italy (lovadina@dimat.unipv.it), Rolf Stenberg, Institute of Mathematics, Helsinki University of Technology, P.O. Box 1500, 02015 HUT, Finland (stenberg@hut.fi).

ISBN 951-22-7281-4

ISSN 0784-3143

HUT Mathematics, Sep 17, 2004

Helsinki University of Technology

Department of Engineering Physics and Mathematics

Institute of Mathematics

P.O. Box 1100, 02015 HUT, Finland

email:math@hut.fi http://www.math.hut.fi/ 
1. Introduction. In this paper we present an a posteriori error analysis for the so-called 'Linked Interpolation Technique' (cf. [2], [3] and [22], for instance) to approximate the solution of the Reissner-Mindlin plate problem.

It is worth noticing that the main effort concerning the finite element discretization of the plate bending problems has been focused on proposing and analyzing locking-free schemes. As a consequence, most of the mathematical literature on the subject is addressed to establish a priori error estimates. We mention here, in a totally non-exhaustive way, the works [1], [5], [7], [13], [16], [19], [21], and the references therein. On the contrary, when considering the a posteriori error analysis for plates, only very few results are available (see [8], [9] and [15]).

In this work we consider the so-called 'Linked Interpolation Technique', focusing on two triangular elements: the first one is the low-order element proposed in [22] (see also [23]), while the second one is the quadratic scheme proposed in [3]. An a priori error analysis has been developed for both the methods in $[17,18]$ and [3], respectively. We also remark that the our a posteriori error analysis may be straightforwardly extended to other schemes taking advantage of the 'Linked Interpolation Technique', such as the quadrilateral elements considered in [2] and [3], for example.

An outline of the paper is as follows. In Section 2 we briefly recall the ReissnerMindlin problem, together with a mixed variational formulation and some useful regularity results. The 'Linked Interpolation Technique' is described in Section 3, where we also develop an a priori analysis, which can be considered as an improvement over the ones detailed in [17] or [18]. Section 4 is devoted to the a posteriori error estimates. In particular we introduce our estimator, and we prove its reliability (Section 4.1) and efficiency (Section 4.2). We point out that in the paper we consider the case of a clamped plate only for simplicity. Indeed, both the a priori and the a posteriori error analysis can be easily adapted to cover other relevant boundary conditions.

Throughout the paper we will use standard notations for Sobolev norms and seminorms. Moreover, we will denote with $C$ a generic constant independent of the mesh parameter $h$ and the plate thickness $t$, which may take different values in different occurrences.

2. The Reissner-Mindlin problem. The Reissner-Mindlin equations for a clamped plate with polygonal mid-plane $\Omega$ require to find $(\boldsymbol{\theta}, w, \gamma)$ such that

$$
\begin{cases}-\operatorname{div} \mathbf{C} \varepsilon(\boldsymbol{\theta})-\boldsymbol{\gamma}=0 & \text { in } \Omega \\ -\operatorname{div} \gamma=g & \text { in } \Omega \\ \boldsymbol{\gamma}=\mu t^{-2}(\boldsymbol{\nabla} w-\boldsymbol{\theta}) & \text { in } \Omega \\ \boldsymbol{\theta}=0, w=0 & \text { on } \partial \Omega\end{cases}
$$

Here, $\mathbf{C}$ is the tensor of bending moduli, $\boldsymbol{\theta}$ represents the rotations, $w$ the transversal displacement, $\gamma$ the scaled shear stresses and $g$ a given transversal load. Moreover, $\varepsilon$ is the usual symmetric gradient operator, $\mu$ is the shear modulus, and $t$ is the thickness. The classical variational formulation of problem (2.1) is

$$
\begin{cases}\text { Find }(\boldsymbol{\theta}, w, \gamma) \in \boldsymbol{\Theta} \times W \times\left(L^{2}(\Omega)\right)^{2}: & \\ a(\boldsymbol{\theta}, \boldsymbol{\eta})+(\boldsymbol{\nabla} v-\boldsymbol{\eta}, \boldsymbol{\gamma})=(g, v) & (\boldsymbol{\eta}, v) \in \boldsymbol{\Theta} \times W \\ (\boldsymbol{\nabla} w-\boldsymbol{\theta}, \boldsymbol{\tau})-\mu^{-1} t^{2}(\boldsymbol{\gamma}, \boldsymbol{\tau})=0 & \boldsymbol{\tau} \in\left(L^{2}(\Omega)\right)^{2},\end{cases}
$$

where $\boldsymbol{\Theta}=\left(H_{0}^{1}(\Omega)\right)^{2}, W=H_{0}^{1}(\Omega),(\cdot, \cdot)$ is the inner-product in $L^{2}(\Omega)$ and

$$
a(\boldsymbol{\theta}, \boldsymbol{\eta}):=\int_{\Omega} \mathbf{C} \varepsilon(\boldsymbol{\theta}): \varepsilon(\boldsymbol{\eta}) .
$$

Following [10], we write the pair $(\boldsymbol{\theta}, w)$ as

$$
(\boldsymbol{\theta}, w)=\left(\boldsymbol{\theta}_{0}+\boldsymbol{\theta}_{r}, w_{0}+w_{r}\right)
$$


where the pair $\left(\boldsymbol{\theta}_{0},, w_{0}\right)$ is the solution of the limit problem:

$$
\begin{cases}\text { Find }\left(\boldsymbol{\theta}_{0}, w_{0}, \boldsymbol{\gamma}_{0}\right) \in \boldsymbol{\Theta} \times W \times \boldsymbol{\Gamma}: & \\ a\left(\boldsymbol{\theta}_{0}, \boldsymbol{\eta}\right)+<\boldsymbol{\nabla} v-\boldsymbol{\eta}, \boldsymbol{\gamma}_{0}>=(g, v) & (\boldsymbol{\eta}, v) \in \boldsymbol{\Theta} \times W, \\ <\boldsymbol{\nabla} w_{0}-\boldsymbol{\theta}_{0}, \boldsymbol{\tau}>=0 & \boldsymbol{\tau} \in \boldsymbol{\Gamma},\end{cases}
$$

and $\left(\boldsymbol{\theta}_{r}, w_{r}\right)$ can be thought as a remainder. Furthermore, $\boldsymbol{\Gamma}=H^{-1}(\operatorname{div}, \Omega)$ and $<\cdot, \cdot\rangle$ is the duality pairing between $H_{0}(\operatorname{rot}, \Omega)$ and $H^{-1}(\operatorname{div}, \Omega)$. One has (cf. [10])

Proposition 2.1. Suppose that $\Omega$ is convex and $g \in L^{2}(\Omega)$. Then it holds

$$
\begin{gathered}
\left\|w_{0}\right\|_{3}+\|\boldsymbol{\theta}\|_{2}+\|\boldsymbol{\gamma}\|_{0}+t\|\gamma\|_{1} \leq C\left(\|g\|_{-1}+t\|g\|_{0}\right), \\
\left\|\boldsymbol{\theta}_{r}\right\|_{1} \leq C t\|g\|_{-1} \\
\left\|w_{r}\right\|_{2} \leq C t\left(\|g\|_{-1}+t\|g\|_{0}\right) .
\end{gathered}
$$

3. The Linked Interpolation Scheme and an a priori analysis. In this Section we present the general idea of the Linked Interpolation Technique (see [3] and [22], for instance), together with two examples of triangular elements. Furthermore, focusing on the lowest-order element, we develop an a priori error analysis which improves the result obtained in [3] and [18].

3.1. The Linked Interpolation Scheme. Let $\left\{\mathcal{T}_{h}\right\}_{h>0}$ be a sequence of decompositions of $\Omega$ into triangular elements $T$, satisfying the usual compatibility conditions (see [12]). We also assume that the family $\left\{\mathcal{T}_{h}\right\}_{h>0}$ is regular, i.e. there exists a constant $\sigma>0$ such that

$$
h_{T} \leq \sigma \rho_{T} \quad \forall T \in \mathcal{T}_{h},
$$

where $h_{T}$ is the diameter of the element $T$ and $\rho_{T}$ is the maximum diameter of the circles contained in $T$. We recall (see [12], for instance) that regularity implies the minimum angle condition: there exists a constant $\alpha>0$ such that

$$
\alpha_{T} \geq \alpha \quad \forall T \in \mathcal{T}_{h},
$$

where $\alpha_{T}$ denotes the smallest inner angle of $T$. Moreover, given the decomposition $\mathcal{T}_{h}$ we will denote with $\mathcal{E}_{h}$ the set of the edges $e$ of the triangles $T \in \mathcal{T}_{h}$. We now select the finite element spaces $\boldsymbol{\Theta}_{h} \subset \boldsymbol{\Theta}, W_{h} \subset W, \boldsymbol{\Gamma}_{h} \subset L^{2}(\Omega)^{2}$, together with a suitable linear operator (the so-called linking operator)

$$
L: \Theta_{h} \longrightarrow H_{0}^{1}(\Omega) .
$$

We then form the following finite dimensional subspace of $\boldsymbol{X}:=\boldsymbol{\Theta} \times W$ :

$$
\boldsymbol{X}_{h}=\left\{\left(\boldsymbol{\eta}_{h}, v_{h}^{*}\right)=\left(\boldsymbol{\eta}_{h}, v_{h}+L \boldsymbol{\eta}_{h}\right): \boldsymbol{\eta}_{h} \in \mathbf{\Theta}_{h}, v_{h} \in W_{h}\right\},
$$

and we finally consider the discrete problem

$$
\begin{cases}\text { Find }\left(\boldsymbol{\theta}_{h}, w_{h}^{*} ; \boldsymbol{\gamma}_{h}\right) \in \boldsymbol{X}_{h} \times \boldsymbol{\Gamma}_{h}: & \\ a\left(\boldsymbol{\theta}_{h}, \boldsymbol{\eta}_{h}\right)+\left(\boldsymbol{\gamma}_{h}, \boldsymbol{\nabla} v_{h}^{*}-\boldsymbol{\eta}_{h}\right)=\left(g, v_{h}^{*}\right) & \left(\boldsymbol{\eta}_{h}, v_{h}^{*}\right) \in \boldsymbol{X}_{h}, \\ \left(\boldsymbol{\nabla} w_{h}^{*}-\boldsymbol{\theta}_{h}, \boldsymbol{\tau}_{h}\right)-\mu^{-1} t^{2}\left(\boldsymbol{\gamma}_{h}, \boldsymbol{\tau}_{h}\right)=0 & \boldsymbol{\tau}_{h} \in \boldsymbol{\Gamma}_{h} .\end{cases}
$$


REMARK 3.1. We point out that eliminating $\gamma_{h}$ from system (3.5), our scheme is equivalent to the following problem involving only the rotations and the vertical displacements:

$$
\left\{\begin{array}{l}
\text { Find }\left(\boldsymbol{\theta}_{h}, w_{h}^{*}\right) \in \boldsymbol{X}_{h}: \\
a\left(\boldsymbol{\theta}_{h}, \boldsymbol{\eta}_{h}\right)+\mu t^{-2}\left(P_{h}\left(\boldsymbol{\nabla} w_{h}^{*}-\boldsymbol{\theta}_{h}\right), P_{h}\left(\boldsymbol{\nabla} v_{h}^{*}-\boldsymbol{\eta}_{h}\right)\right)=\left(g, v_{h}\right) \quad \forall\left(\boldsymbol{\eta}_{h}, v_{h}^{*}\right) \in \boldsymbol{X}_{h},
\end{array}\right.
$$

where $P_{h}$ denotes the $L^{2}$-projection operator onto $\boldsymbol{\Gamma}_{h}$.

We are now ready to present the following two elements (for other methods based on the same strategy, see e.g. [2, 3]).

3.1.1. The linear element. This element (see [22]) is described by the finite element spaces

$$
\begin{gathered}
\boldsymbol{\Theta}_{h}=\left\{\boldsymbol{\eta} \in \boldsymbol{\Theta}: \boldsymbol{\eta}_{\mid T} \in\left(P_{1}(T) \oplus B_{3}(T)\right)^{2}\right\}, \\
W_{h}=\left\{v \in W: v_{\mid T} \in P_{1}(T)\right\}, \\
\boldsymbol{\Gamma}_{h}=\left\{\boldsymbol{\tau} \in L^{2}(\Omega)^{2}: \boldsymbol{\tau}_{\mid T} \in P_{0}(T)^{2}\right\},
\end{gathered}
$$

where $P_{k}(T)$ is the space of polynomials of degree at most $k$ defined on $T$ and $B_{3}(T)=$ $P_{3}(T) \cap H_{0}^{1}(T)$ is the space of cubic bubbles on $T$. The linking operator $L: \boldsymbol{\Theta}_{h} \longrightarrow$ $H_{0}^{1}(\Omega)$ is defined as follows. For each $T \in \mathcal{T}_{h}$, we set

$$
\varphi_{i}=\lambda_{j} \lambda_{k} \quad \text { and } \quad E B_{2}(T)=\operatorname{Span}\left\{\varphi_{i}\right\}_{1 \leq i \leq 3},
$$

where $\left\{\lambda_{i}\right\}_{1 \leq i \leq 3}$ are the barycentric coordinates of the triangle $T$ and the indices $(i, j, k)$ form a permutation of the set $(1,2,3)$. Then, the operator $L$ is locally defined as

$$
L \boldsymbol{\eta}_{h \mid T}=\sum_{i=1}^{3} \alpha_{i} \varphi_{i} \in E B_{2}(T)
$$

where the coefficients $\alpha_{i}$ are determined by requiring that

$$
\left(\boldsymbol{\nabla} L \boldsymbol{\eta}_{h}-\boldsymbol{\eta}_{h}\right) \cdot \mathbf{t} \quad \text { is constant on each } e .
$$

Above, $\mathbf{t}$ denotes the tangential vector to the edge $e$. We recall that for the linking operator it holds (see [17] and [18])

$$
\|\left.\nabla L \boldsymbol{\eta}_{h}\right|_{0, T} \leq C h_{T}\left|\boldsymbol{\eta}_{h}\right|_{1, T}
$$

3.1.2. The quadratic element. This element (see [3]) is described by the finite element spaces

$$
\begin{gathered}
\boldsymbol{\Theta}_{h}=\left\{\boldsymbol{\eta} \in \boldsymbol{\Theta}: \boldsymbol{\eta}_{\mid T} \in P_{2}(T)^{2} \oplus\left(P_{0}(T)^{2} \oplus \nabla B_{3}(T)\right) b_{T}\right\}, \\
W_{h}=\left\{v \in W: v_{\mid T} \in P_{2}(T) \oplus B_{3}(T)\right\}, \\
\boldsymbol{\Gamma}_{h}=\left\{\boldsymbol{\tau} \in L^{2}(\Omega)^{2}: \boldsymbol{\tau}_{\mid T} \in P_{0}(T)^{2} \oplus \nabla B_{3}(T)\right\},
\end{gathered}
$$

where $b_{T}=27 \lambda_{1} \lambda_{2} \lambda_{3}$. The linking operator $L: \boldsymbol{\Theta}_{h} \longrightarrow H_{0}^{1}(\Omega)$ is defined as follows. For each $T \in \mathcal{T}_{h}$, we set

$$
\varphi_{i}=\lambda_{j} \lambda_{k}\left(\lambda_{k}-\lambda_{j}\right) \quad \text { and } \quad E B_{3}(T)=\operatorname{Span}\left\{\varphi_{i}\right\}_{1 \leq i \leq 3}
$$


where the indices $(i, j, k)$ form a permutation of the set $(1,2,3)$. Then, the operator $L$ is locally defined as

$$
L \boldsymbol{\eta}_{h \mid T}=\sum_{i=1}^{3} \alpha_{i} \varphi_{i} \in E B_{3}(T),
$$

where the coefficients $\alpha_{i}$ 's are determined by requiring that

$$
\left(\boldsymbol{\nabla} L \boldsymbol{\eta}_{h}-\boldsymbol{\eta}_{h}\right) \cdot \mathbf{t} \quad \text { is linear on each } e .
$$

For this linking operator it holds (see [3])

$$
\|\left.\boldsymbol{\nabla} L \boldsymbol{\eta}_{h}\right|_{0, T} \leq C h_{T}^{2}\left|\boldsymbol{\eta}_{h}\right|_{2, T} \leq C h_{T}\left|\boldsymbol{\eta}_{h}\right|_{1, T}
$$

3.2. A priori error estimates. In this section we focus on the lowest-order element detailed in Section 3.1.1, but a similar technique (together with the ideas developed in [19]) may be applied to appropriately treat the higher-order case of Section 3.1.2. Following the lines of $[10,17,19,21]$, we prove a priori error estimates with respect to the norms

$$
\|(\boldsymbol{\eta}, v)\|_{h}^{2}:=\|\boldsymbol{\eta}\|_{1}^{2}+\|v\|_{1}^{2}+\sum_{T \in \mathcal{T}_{h}} \frac{1}{h_{T}^{2}+t^{2}}\|\boldsymbol{\nabla} v-\boldsymbol{\eta}\|_{0, T}^{2} \quad \forall(\boldsymbol{\eta}, v) \in \mathbf{\Theta} \times W
$$

and

$$
\|\boldsymbol{\tau}\|_{-1}+t\|\boldsymbol{\tau}\|_{0} \quad \forall \boldsymbol{\tau} \in L^{2}(\Omega)^{2} .
$$

We will also use the following discrete norm

$$
\|\boldsymbol{\tau}\|_{h}^{2}:=\sum_{T \in \mathcal{T}_{h}} h_{T}^{2}\|\boldsymbol{\tau}\|_{0, T}^{2}+t^{2}\|\boldsymbol{\tau}\|_{0}^{2} \quad \forall \boldsymbol{\tau} \in L^{2}(\Omega)^{2} .
$$

Before proceeding, we need the following lemma, which establishes a suitable norm equivalence in the used finite element spaces.

Lemma 3.1. Consider the finite element spaces and the linking operator detailed in Section 3.1.1, and let $P_{h}$ denote the $L^{2}$-projection operator on $\boldsymbol{\Gamma}_{h}$. Then for each $\left(\boldsymbol{\eta}_{h}, v_{h}^{*}\right) \in \boldsymbol{X}_{h}$ it holds

$$
\left(\left\|\boldsymbol{\eta}_{h}\right\|_{1}^{2}+\sum_{T \in \mathcal{T}_{h}} \frac{1}{h_{T}^{2}+t^{2}}\left\|P_{h}\left(\boldsymbol{\nabla} v_{h}^{*}-\boldsymbol{\eta}_{h}\right)\right\|_{0, T}^{2}\right)^{1 / 2} \leq\left\|\left(\boldsymbol{\eta}_{h}, v_{h}^{*}\right)\right\| h
$$

and

$$
\left\|\left(\boldsymbol{\eta}_{h}, v_{h}^{*}\right)\right\|_{h} \leq C\left(\left\|\boldsymbol{\eta}_{h}\right\|_{1}^{2}+\sum_{T \in \mathcal{T}_{h}} \frac{1}{h_{T}^{2}+t^{2}}\left\|P_{h}\left(\boldsymbol{\nabla} v_{h}^{*}-\boldsymbol{\eta}_{h}\right)\right\|_{0, T}^{2}\right)^{1 / 2} .
$$

Proof. Since (3.24) is trivial, we only consider (3.25). Therefore, take $\boldsymbol{\eta}_{h} \in \mathbf{\Theta}_{h}$, $v_{h} \in W_{h}$ and form $\left(\boldsymbol{\eta}_{h}, v_{h}^{*}\right)=\left(\boldsymbol{\eta}_{h}, v_{h}+L \boldsymbol{\eta}_{h}\right) \in \boldsymbol{X}_{h}$. We first notice that

$$
\begin{aligned}
\left\|\boldsymbol{\nabla} v_{h}^{*}\right\|_{0}^{2} & \leq 2\left(\left\|\boldsymbol{\nabla} v_{h}^{*}-\boldsymbol{\eta}_{h}\right\|_{0}^{2}+\left\|\boldsymbol{\eta}_{h}\right\|_{0}^{2}\right) \\
& \leq C\left(\sum_{T \in \mathcal{T}_{h}} \frac{1}{h_{T}^{2}+t^{2}}\left\|\boldsymbol{\nabla} v_{h}^{*}-\boldsymbol{\eta}_{h}\right\|_{0, T}^{2}+\left\|\boldsymbol{\eta}_{h}\right\|_{1}^{2}\right),
\end{aligned}
$$

so that, by Poincarè's inequality, we have

$$
\left\|v_{h}^{*}\right\|_{1}^{2} \leq C\left(\sum_{T \in \mathcal{T}_{h}} \frac{1}{h_{T}^{2}+t^{2}}\left\|\nabla v_{h}^{*}-\boldsymbol{\eta}_{h}\right\|_{0, T}^{2}+\left\|\boldsymbol{\eta}_{h}\right\|_{1}^{2}\right),
$$


Next, we write $\boldsymbol{\nabla} v_{h}^{*}-\boldsymbol{\eta}_{h}$ as

$$
\begin{aligned}
\boldsymbol{\nabla} v_{h}^{*}-\boldsymbol{\eta}_{h} & =\boldsymbol{\nabla} v_{h}+\boldsymbol{\nabla} L \boldsymbol{\eta}_{h}-\boldsymbol{\eta}_{h}=P_{h} \boldsymbol{\nabla} v_{h}+\boldsymbol{\nabla} L \boldsymbol{\eta}_{h}-\boldsymbol{\eta}_{h} \\
& =P_{h} \boldsymbol{\nabla} v_{h}^{*}-\left(P_{h} \boldsymbol{\nabla} L \boldsymbol{\eta}_{h}-\boldsymbol{\nabla} L \boldsymbol{\eta}_{h}\right)-\boldsymbol{\eta}_{h} \\
& =P_{h}\left(\boldsymbol{\nabla} v_{h}^{*}-\boldsymbol{\eta}_{h}\right)-\left(P_{h} \boldsymbol{\nabla} L \boldsymbol{\eta}_{h}-\boldsymbol{\nabla} L \boldsymbol{\eta}_{h}\right)+\left(P_{h} \boldsymbol{\eta}_{h}-\boldsymbol{\eta}_{h}\right) .
\end{aligned}
$$

Therefore, we have

$$
\begin{aligned}
\left\|\boldsymbol{\nabla} v_{h}^{*}-\boldsymbol{\eta}_{h}\right\|_{0, T} & \leq\left\|P_{h}\left(\boldsymbol{\nabla} v_{h}^{*}-\boldsymbol{\eta}_{h}\right)\right\|_{0, T} \\
& +\left\|P_{h} \boldsymbol{\nabla} L \boldsymbol{\eta}_{h}-\nabla L \boldsymbol{\eta}_{h}\right\|_{0, T}+\left\|P_{h} \boldsymbol{\eta}_{h}-\boldsymbol{\eta}_{h}\right\|_{0, T} .
\end{aligned}
$$

Since (see also (3.13))

$$
\left\|P_{h} \boldsymbol{\nabla} L \boldsymbol{\eta}_{h}-\left.\nabla L \boldsymbol{\eta}_{h}\right|_{0, T} \leq 2|| \nabla L \boldsymbol{\eta}_{h}\right\|_{0, T} \leq C h_{T}\left|\boldsymbol{\eta}_{h}\right|_{1, T}
$$

and

$$
\left\|P_{h} \boldsymbol{\eta}_{h}-\boldsymbol{\eta}_{h}\right\|_{0, T} \leq C h_{T}\left|\boldsymbol{\eta}_{h}\right|_{1, T}
$$

from (3.29) we obtain

$$
\begin{aligned}
\frac{1}{h_{T}^{2}+t^{2}}\left\|\boldsymbol{\nabla} v_{h}^{*}-\boldsymbol{\eta}_{h}\right\|_{0, T}^{2} & \leq C\left(\frac{1}{h_{T}^{2}+t^{2}}\left\|P_{h}\left(\nabla v_{h}^{*}-\boldsymbol{\eta}_{h}\right)\right\|_{0, T}^{2}+\frac{h_{T}^{2}}{h_{T}^{2}+t^{2}}\left|\boldsymbol{\eta}_{h}\right|_{1, T}^{2}\right) \\
& \leq C\left(\frac{1}{h_{T}^{2}+t^{2}}\left\|P_{h}\left(\boldsymbol{\nabla} v_{h}^{*}-\boldsymbol{\eta}_{h}\right)\right\|_{0, T}^{2}+\left|\boldsymbol{\eta}_{h}\right|_{1, T}^{2}\right) .
\end{aligned}
$$

Therefore, we get

$$
\sum_{T \in \mathcal{T}_{h}} \frac{1}{h_{T}^{2}+t^{2}}\left\|\boldsymbol{\nabla} v_{h}^{*}-\boldsymbol{\eta}_{h}\right\|_{0, T}^{2} \leq C\left(\sum_{T \in \mathcal{T}_{h}} \frac{1}{h_{T}^{2}+t^{2}}\left\|P_{h}\left(\boldsymbol{\nabla} v_{h}^{*}-\boldsymbol{\eta}_{h}\right)\right\|_{0, T}^{2}+\left\|\boldsymbol{\eta}_{h}\right\|_{1}^{2}\right)
$$

Using (3.27) and (3.31) we deduce estimate (3.25).

It is now useful to set

$$
\begin{aligned}
\mathcal{A}(\boldsymbol{\theta}, w, \boldsymbol{\gamma} ; \boldsymbol{\eta}, v, \boldsymbol{\tau}):= & a(\boldsymbol{\theta}, \boldsymbol{\eta})+(\boldsymbol{\nabla} v-\boldsymbol{\eta}, \boldsymbol{\gamma}) \\
& -(\boldsymbol{\nabla} w-\boldsymbol{\theta}, \boldsymbol{\tau})+\mu^{-1} t^{2}(\boldsymbol{\gamma}, \boldsymbol{\tau})
\end{aligned}
$$

Therefore, the continuous problem (2.2) reads

$$
\left\{\begin{array}{l}
\text { Find }(\boldsymbol{\theta}, w ; \boldsymbol{\gamma}) \in \boldsymbol{X} \times L^{2}(\Omega)^{2} \text { s.t. } \\
\mathcal{A}(\boldsymbol{\theta}, w, \boldsymbol{\gamma} ; \boldsymbol{\eta}, v, \boldsymbol{\tau})=(g, v) \quad \forall(\boldsymbol{\eta}, v ; \boldsymbol{\tau}) \in \boldsymbol{X} \times L^{2}(\Omega)^{2}
\end{array}\right.
$$

while the discrete problem (3.5) is

$$
\left\{\begin{array}{l}
\text { Find }\left(\boldsymbol{\theta}_{h}, w_{h}^{*} ; \boldsymbol{\gamma}_{h}\right) \in \boldsymbol{X}_{h} \times \boldsymbol{\Gamma}_{h} \text { s.t. } \\
\mathcal{A}\left(\boldsymbol{\theta}_{h}, w_{h}^{*}, \boldsymbol{\gamma}_{h} ; \boldsymbol{\eta}_{h}, v_{h}^{*}, \boldsymbol{\tau}_{h}\right)=\left(g, v_{h}^{*}\right) \quad \forall\left(\boldsymbol{\eta}_{h}, v_{h}^{*} ; \boldsymbol{\tau}_{h}\right) \in \boldsymbol{X}_{h} \times \boldsymbol{\Gamma}_{h} .
\end{array}\right.
$$

We have the following stability result, for which we only sketch the proof, since it takes advantage of the same techniques detailed in [10] and [17].

Proposition 3.2. Given $\left(\boldsymbol{\beta}_{h}, z_{h}^{*} ; \boldsymbol{\rho}_{h}\right) \in \boldsymbol{X}_{h} \times \boldsymbol{\Gamma}_{h}$ there exists $\left(\boldsymbol{\eta}_{h}, v_{h}^{*} ; \boldsymbol{\tau}_{h}\right) \in$ $\boldsymbol{X}_{h} \times \boldsymbol{\Gamma}_{h}$ such that

$$
\mathcal{A}\left(\boldsymbol{\beta}_{h}, z_{h}^{*}, \boldsymbol{\rho}_{h} ; \boldsymbol{\eta}_{h}, v_{h}^{*}, \boldsymbol{\tau}_{h}\right) \geq C\left(\left\|\left(\boldsymbol{\beta}_{h}, z_{h}^{*}\right)\right\|_{h}^{2}+\left\|\boldsymbol{\rho}_{h}\right\|_{-1}^{2}+t^{2}\left\|\boldsymbol{\rho}_{h}\right\|_{0}^{2}\right)
$$




$$
\left\|\left(\boldsymbol{\eta}_{h}, v_{h}^{*}\right)\right\|_{h}+\left\|\boldsymbol{\tau}_{h}\right\|_{-1}+t\left\|\boldsymbol{\tau}_{h}\right\|_{0} \leq C\left(\left\|\left(\boldsymbol{\beta}_{h}, z_{h}^{*}\right)\right\|_{h}+\left\|\boldsymbol{\rho}_{h}\right\|_{-1}+t\left\|\boldsymbol{\rho}_{h}\right\|_{0}\right)
$$

Proof. Let us $\left(\boldsymbol{\beta}_{h}, z_{h}^{*} ; \boldsymbol{\rho}_{h}\right)$ be given in $\boldsymbol{X}_{h} \times \boldsymbol{\Gamma}_{h}$. Using exactly the same arguments of [10] and [17] we get that there exists $\left(\boldsymbol{\eta}_{h}, v_{h}^{*} ; \boldsymbol{\tau}_{h}\right)$ in $\boldsymbol{X}_{h} \times \boldsymbol{\Gamma}_{h}$ such that

$$
\mathcal{A}\left(\boldsymbol{\beta}_{h}, z_{h}^{*}, \boldsymbol{\rho}_{h} ; \boldsymbol{\eta}_{h}, v_{h}^{*}, \boldsymbol{\tau}_{h}\right) \geq C\left(\left\|\boldsymbol{\beta}_{h}\right\|_{1}^{2}+\sum_{T \in \mathcal{T}_{h}} \frac{1}{h_{T}^{2}+t^{2}}\left\|P_{h}\left(\boldsymbol{\nabla} z_{h}^{*}-\boldsymbol{\beta}_{h}\right)\right\|_{0, T}^{2}+\left\|\boldsymbol{\rho}_{h}\right\|_{h}^{2}\right)
$$

and

$$
\begin{aligned}
\left\|\boldsymbol{\eta}_{h}\right\|_{1}+ & \left(\sum_{T \in \mathcal{T}_{h}} \frac{1}{h_{T}^{2}+t^{2}}\left\|P_{h}\left(\boldsymbol{\nabla} v_{h}^{*}-\boldsymbol{\eta}_{h}\right)\right\|_{0, T}^{2}\right)^{1 / 2}+\left\|\boldsymbol{\tau}_{h}\right\|_{h} \\
& \leq C\left(\left\|\boldsymbol{\beta}_{h}\right\|_{1}+\left(\sum_{T \in \mathcal{T}_{h}} \frac{1}{h_{T}^{2}+t^{2}}\left\|P_{h}\left(\boldsymbol{\nabla} z_{h}^{*}-\boldsymbol{\beta}_{h}\right)\right\|_{0, T}^{2}\right)^{1 / 2}+\left\|\boldsymbol{\rho}_{h}\right\|_{h}\right) .
\end{aligned}
$$

We now use Lemma 3.1 to infer that given $\left(\boldsymbol{\beta}_{h}, z_{h}^{*} ; \boldsymbol{\rho}_{h}\right) \in \boldsymbol{X}_{h} \times \boldsymbol{\Gamma}_{h}$, there exists $\left(\boldsymbol{\eta}_{h}, v_{h}^{*} ; \boldsymbol{\tau}_{h}\right) \in \boldsymbol{X}_{h} \times \boldsymbol{\Gamma}_{h}$ such that

$$
\mathcal{A}\left(\boldsymbol{\beta}_{h}, z_{h}^{*}, \boldsymbol{\rho}_{h} ; \boldsymbol{\eta}_{h}, v_{h}^{*}, \boldsymbol{\tau}_{h}\right) \geq C\left(\left\|\left(\boldsymbol{\beta}_{h}, v_{h}^{*}\right)\right\|_{h}^{2}+\left\|\boldsymbol{\rho}_{h}\right\|_{h}^{2}\right)
$$

and

$$
\left\|\left(\boldsymbol{\eta}_{h}, v_{h}^{*}\right)\right\|_{h}+\left\|\boldsymbol{\tau}_{h}\right\|_{h} \leq C\left(\left\|\left(\boldsymbol{\beta}_{h}, z_{h}^{*}\right)\right\|_{h}+\left\|\boldsymbol{\rho}_{h}\right\|_{h}\right) .
$$

Stability with respect to the shear norm detailed in (3.22) is finally obtained by using the 'Pitkäranta-Verfürth trick' (cf. [20], [24] and also [11]).

We now prove an error estimate, which can be considered as an improvement of the ones obtained in [18] and [17].

Proposition 3.3. Suppose that $\Omega$ is a convex polygon and $g \in L^{2}(\Omega)$ and consider the element detailed in Section 3.1.1. Let $(\boldsymbol{\theta}, w ; \gamma) \in \boldsymbol{X} \times L^{2}(\Omega)^{2}$ and $\left(\boldsymbol{\theta}_{h}, w_{h}^{*} ; \boldsymbol{\gamma}_{h}\right) \in \boldsymbol{X}_{h} \times \boldsymbol{\Gamma}_{h}$ be the solutions of problem (3.35) and (3.36), respectively. Then the following a priori estimates holds

$$
\left\|\left(\boldsymbol{\theta}-\boldsymbol{\theta}_{h}, w-w_{h}^{*}\right)\right\|_{h}+\left\|\boldsymbol{\gamma}-\gamma_{h}\right\|_{-1}+t\left\|\boldsymbol{\gamma}-\gamma_{h}\right\|_{0} \leq C h\left(\|g\|_{-1}+t\|g\|_{0}\right) .
$$

Proof. Since our method is consistent (cf. (3.35) and (3.36)) and stable (see Proposition 3.2 ), error estimates with respect to the norms in question can be established in the standard way. Hence, let

$$
\left(\boldsymbol{\theta}_{I}, w_{I}^{*} ; \boldsymbol{\gamma}_{I}\right)=\left(\boldsymbol{\theta}_{I}, w_{I}+L \boldsymbol{\theta}_{I} ; \boldsymbol{\gamma}_{I}\right) \in \boldsymbol{X}_{h} \times \boldsymbol{\Gamma}_{h}
$$

be a suitable interpolant (to be specified later) of the continuous solution $\left(\boldsymbol{\theta}, w^{*} ; \boldsymbol{\gamma}\right)$. Corresponding to $\left(\boldsymbol{\theta}_{h}-\boldsymbol{\theta}_{I}, w_{h}^{*}-w_{I}^{*} ; \boldsymbol{\gamma}_{h}-\boldsymbol{\gamma}_{I}\right) \in \boldsymbol{X}_{h} \times \boldsymbol{\Gamma}_{h}$ there exists (see Proposition 3.2) $\left(\boldsymbol{\eta}_{h}, v_{h}^{*} ; \boldsymbol{\tau}_{h}\right) \in \boldsymbol{X}_{h} \times \boldsymbol{\Gamma}_{h}$ such that

$$
\begin{gathered}
\mathcal{A}\left(\boldsymbol{\theta}_{h}-\boldsymbol{\theta}_{I}, w_{h}^{*}-w_{I}^{*}, \gamma_{h}-\gamma_{I} ; \boldsymbol{\eta}_{h}, v_{h}^{*}, \boldsymbol{\tau}_{h}\right) \geq C\left(\left\|\left(\boldsymbol{\theta}_{h}-\boldsymbol{\theta}_{I}, w_{h}^{*}-w_{I}^{*}\right)\right\|_{h}^{2}\right. \\
\left.+\left\|\gamma_{h}-\gamma_{I}\right\|_{-1}^{2}+t^{2}\left\|\gamma_{h}-\gamma_{I}\right\|_{0}^{2}\right),
\end{gathered}
$$

and

$$
\begin{aligned}
\left\|\left(\boldsymbol{\eta}_{h}, v_{h}^{*}\right)\right\|_{h}+\left\|\boldsymbol{\tau}_{h}\right\|_{-1}+t\left\|\boldsymbol{\tau}_{h}\right\|_{0} \\
\quad \leq C\left(\left\|\left(\boldsymbol{\theta}_{h}-\boldsymbol{\theta}_{I}, w_{h}^{*}-w_{I}^{*}\right)\right\|_{h}+\left\|\gamma_{h}-\gamma_{I}\right\|_{-1}+t\left\|\gamma_{h}-\gamma_{I}\right\|_{0}\right) .
\end{aligned}
$$


By consistency it holds

$$
\begin{aligned}
\mathcal{A}\left(\boldsymbol{\theta}_{h}-\boldsymbol{\theta}_{I}, w_{h}^{*}-w_{I}^{*}, \boldsymbol{\gamma}_{h}-\gamma_{I} ; \boldsymbol{\eta}_{h}, v_{h}^{*}, \boldsymbol{\tau}_{h}\right)=\mathcal{A}\left(\boldsymbol{\theta}-\boldsymbol{\theta}_{I}, w-w_{I}^{*}, \boldsymbol{\gamma}-\gamma_{I} ; \boldsymbol{\eta}_{h}, v_{h}^{*}, \boldsymbol{\tau}_{h}\right) \\
=a\left(\boldsymbol{\theta}-\boldsymbol{\theta}_{I}, \boldsymbol{\eta}_{h}\right)+\left(\boldsymbol{\nabla} v_{h}^{*}-\boldsymbol{\eta}_{h}, \boldsymbol{\gamma}-\gamma_{I}\right) \\
-\left(\boldsymbol{\nabla}\left(w-w_{I}^{*}\right)-\left(\boldsymbol{\theta}-\boldsymbol{\theta}_{I}\right), \boldsymbol{\tau}_{h}\right)+\mu^{-1} t^{2}\left(\boldsymbol{\gamma}-\boldsymbol{\gamma}_{I}, \boldsymbol{\tau}_{h}\right) \\
=(I)+(I I)+(I I I)+(I V) .
\end{aligned}
$$

To bound the four terms above, we first choose the interpolants $\boldsymbol{\theta}_{I}, w_{I}^{*}$ and $\gamma_{I}$ as follows. According to the splitting (2.3), $\boldsymbol{\theta}_{I}$ is given by

$$
\boldsymbol{\theta}_{I}:=\mathcal{I} \boldsymbol{\theta}=\mathcal{I} \boldsymbol{\theta}_{0}+\mathcal{I} \boldsymbol{\theta}_{r},
$$

where $\mathcal{I}$ is the Lagrange interpolating operator. To define $w_{I}^{*}$, we need to specify $w_{I}$ (cf. (3.44)). Again, the splitting (2.3) suggests to set

$$
w_{I}:=\mathcal{I} w=\mathcal{I} w_{0}+\mathcal{I} w_{r} .
$$

Therefore, $w_{I}^{*}$ turns out to be $w_{I}^{*}=w_{I}+L \boldsymbol{\theta}_{I}=\mathcal{I} w+L(\mathcal{I} \boldsymbol{\theta})$. Finally, $\gamma_{I}$ is simply the $L^{2}$-projection of $\gamma$ onto $\boldsymbol{\Gamma}_{h}$.

Estimate for $(I)$. Using the $H^{1}$-continuity of the bilinear form $a(\cdot, \cdot)$, standard approximation results and estimate (2.5) we have

$$
(I)=a\left(\boldsymbol{\theta}-\boldsymbol{\theta}_{I}, \boldsymbol{\eta}_{h}\right) \leq C h\|\boldsymbol{\theta}\|_{2}\left\|\boldsymbol{\eta}_{h}\right\|_{1} \leq C h\left(\|g\|_{-1}+t\|g\|_{0}\right)\left\|\boldsymbol{\eta}_{h}\right\|_{1} .
$$

Estimate for (II). We notice that

$$
\begin{aligned}
(I I)= & \left(\boldsymbol{\nabla} v_{h}^{*}-\boldsymbol{\eta}_{h}, \boldsymbol{\gamma}-\boldsymbol{\gamma}_{I}\right) \\
& \leq\left(\sum_{T \in \mathcal{T}_{h}} \frac{1}{h_{T}^{2}+t^{2}}\left\|\boldsymbol{\nabla} v_{h}^{*}-\boldsymbol{\eta}_{h}\right\|_{0, T}^{2}\right)^{1 / 2}\left(\sum_{T \in \mathcal{T}_{h}}\left(h_{T}^{2}+t^{2}\right)\left\|\boldsymbol{\gamma}-\gamma_{I}\right\|_{0, T}^{2}\right)^{1 / 2},
\end{aligned}
$$

by which, using again (2.5) and standard approximation estimates, we get

$$
(I I) \leq C h\left(\|g\|_{-1}+t\|g\|_{0}\right)\left(\sum_{T \in \mathcal{T}_{h}} \frac{1}{h_{T}^{2}+t^{2}}\left\|\nabla v_{h}^{*}-\boldsymbol{\eta}_{h}\right\|_{0, T}^{2}\right)^{1 / 2} .
$$

Estimate for $(I I I)$.

$$
\begin{aligned}
& (I I I)=-\left(\boldsymbol{\nabla}\left(w-w_{I}^{*}\right)-\left(\boldsymbol{\theta}-\boldsymbol{\theta}_{I}\right), \boldsymbol{\tau}_{h}\right) \\
& \quad \leq\left(\sum_{T \in \mathcal{T}_{h}} \frac{1}{h_{T}^{2}+t^{2}}\left\|\boldsymbol{\nabla}\left(w-w_{I}^{*}\right)-\left(\boldsymbol{\theta}-\boldsymbol{\theta}_{I}\right)\right\|_{0, T}^{2}\right)^{1 / 2}\left(\sum_{T \in \mathcal{T}_{h}}\left(h_{T}^{2}+t^{2}\right)\left\|\boldsymbol{\tau}_{h}\right\|_{0, T}^{2}\right)^{1 / 2} .
\end{aligned}
$$

We now notice that we have (see (2.3), (3.44) and (3.48)-(3.49))

$$
\begin{aligned}
\boldsymbol{\nabla}\left(w-w_{I}^{*}\right)-\left(\boldsymbol{\theta}-\boldsymbol{\theta}_{I}\right) & =\left\{\boldsymbol{\nabla}\left(w_{0}-\mathcal{I} w_{0}-L\left(\mathcal{I} \boldsymbol{\theta}_{0}\right)\right)-\left(\boldsymbol{\theta}_{0}-\mathcal{I} \boldsymbol{\theta}_{0}\right)\right\} \\
& +\left\{\boldsymbol{\nabla}\left(w_{r}-\mathcal{I} w_{r}-L\left(\mathcal{I} \boldsymbol{\theta}_{r}\right)\right)-\left(\boldsymbol{\theta}_{r}-\mathcal{I} \boldsymbol{\theta}_{r}\right)\right\}
\end{aligned}
$$

In [17] it has been proved that

$$
\left|\nabla\left(w_{0}-\mathcal{I} w_{0}-L\left(\mathcal{I} \boldsymbol{\theta}_{0}\right)\right)\right|_{0, T} \leq C h_{T}^{2}\left|w_{0}\right|_{3, T},
$$


while standard approximation results give

$$
\begin{gathered}
\left|\boldsymbol{\theta}_{0}-\mathcal{I} \boldsymbol{\theta}_{0}\right|_{0, T} \leq C h_{T}^{2}\left|\boldsymbol{\theta}_{0}\right|_{2, T} \\
\left|\boldsymbol{\theta}_{r}-\mathcal{I} \boldsymbol{\theta}_{r}\right|_{0, T} \leq C h_{T}^{2}\left|\boldsymbol{\theta}_{r}\right|_{2, T} .
\end{gathered}
$$

Furthermore, using also (3.13) it holds

$$
\begin{aligned}
\mid \boldsymbol{\nabla}\left(w_{r}-\mathcal{I} w_{r}\right. & \left.-L\left(\mathcal{I} \boldsymbol{\theta}_{r}\right)\right)\left.\right|_{0, T} \leq\left|\boldsymbol{\nabla}\left(w_{r}-\mathcal{I} w_{r}\right)\right|_{0, T}+\left|\nabla L\left(\mathcal{I} \boldsymbol{\theta}_{r}\right)\right|_{0, T} \\
& \leq\left|\boldsymbol{\nabla}\left(w_{r}-\mathcal{I} w_{r}\right)\right|_{0, T}+\left|\boldsymbol{\nabla} L\left(\mathcal{I} \boldsymbol{\theta}_{r}-\boldsymbol{\theta}_{r}\right)\right|_{0, T}+\left|\nabla L\left(\boldsymbol{\theta}_{r}\right)\right|_{0, T} \\
& \leq C\left(h_{T}\left|w_{r}\right|_{2, T}+h_{T}\left|\mathcal{I} \boldsymbol{\theta}_{r}-\boldsymbol{\theta}_{r}\right|_{1, T}+h_{T}\left|\boldsymbol{\theta}_{r}\right|_{1, T}\right) \\
& \leq C\left(h_{T}\left|w_{r}\right|_{2, T}+h_{T}^{2}\left|\boldsymbol{\theta}_{r}\right|_{2, T}+h_{T}\left|\boldsymbol{\theta}_{r}\right|_{1, T}\right)
\end{aligned}
$$

From (3.54)-(3.58) we obtain

$$
\begin{aligned}
\sum_{T \in \mathcal{T}_{h}} \frac{1}{h_{T}^{2}+t^{2}} & \left\|\boldsymbol{\nabla}\left(w-w_{I}^{*}\right)-\left(\boldsymbol{\theta}-\boldsymbol{\theta}_{I}\right)\right\|_{0, T}^{2} \\
& \leq C \sum_{T \in \mathcal{T}_{h}} \frac{1}{h_{T}^{2}+t^{2}}\left(h_{T}^{4}\left|w_{0}\right|_{3, T}^{2}+h_{T}^{4}|\boldsymbol{\theta}|_{2, T}^{2}+h_{T}^{2}\left|w_{r}\right|_{2, T}^{2}+h_{T}^{2}\left|\boldsymbol{\theta}_{r}\right|_{1, T}^{2}\right) \\
& \leq C h^{2}\left(\left|w_{0}\right|_{3}^{2}+|\boldsymbol{\theta}|_{2}^{2}\right)+\sum_{T \in \mathcal{T}_{h}} \frac{h_{T}^{2}}{h_{T}^{2}+t^{2}}\left(\left|w_{r}\right|_{2, T}^{2}+\left|\boldsymbol{\theta}_{r}\right|_{1, T}^{2}\right) \\
& \leq C h^{2}\left(\left|w_{0}\right|_{3}^{2}+|\boldsymbol{\theta}|_{2}^{2}\right)+\sum_{T \in \mathcal{T}_{h}} h_{T}^{2}\left(\frac{\left|w_{r}\right|_{2, T}^{2}}{t^{2}}+\frac{\left|\boldsymbol{\theta}_{r}\right|_{1, T}^{2}}{t^{2}}\right) \\
& \leq C h^{2}\left(\left|w_{0}\right|_{3}^{2}+|\boldsymbol{\theta}|_{2}^{2}+\frac{\left|w_{r}\right|_{2}^{2}}{t^{2}}+\frac{\left|\boldsymbol{\theta}_{r}\right|_{1}^{2}}{t^{2}}\right) .
\end{aligned}
$$

Using (2.5)-(2.7), from (3.59) it follows that

$$
\begin{gathered}
\left(\sum_{T \in \mathcal{T}_{h}} \frac{1}{h_{T}^{2}+t^{2}}\left\|\boldsymbol{\nabla}\left(w-w_{I}^{*}\right)-\left(\boldsymbol{\theta}-\boldsymbol{\theta}_{I}\right)\right\|_{0, T}^{2}\right)^{1 / 2} \\
\leq C h\left(\left\|w_{0}\right\|_{3}+\|\boldsymbol{\theta}\|_{2}+\frac{\left\|w_{r}\right\|_{2}}{t}+\frac{\left\|\boldsymbol{\theta}_{r}\right\|_{1}}{t}\right) \\
\leq C h\left(\|g\|_{-1}+t\|g\|_{0}\right) .
\end{gathered}
$$

Therefore, we obtain (see (3.53))

$$
(I I I) \leq C h\left(\|g\|_{-1}+t\|g\|_{0}\right)\left(\sum_{T \in \mathcal{T}_{h}}\left(h_{T}^{2}+t^{2}\right)\left\|\boldsymbol{\tau}_{h}\right\|_{0, T}^{2}\right)^{1 / 2} .
$$

Estimate for $(I V)$. We simply notice that

$$
(I V)=\mu^{-1} t^{2}\left(\boldsymbol{\gamma}-\gamma_{I}, \boldsymbol{\tau}_{h}\right) \leq C t\left\|\boldsymbol{\gamma}-\gamma_{I}\right\|_{0} t\left\|\boldsymbol{\tau}_{h}\right\|_{0} \leq C h\left(\|g\|_{-1}+t\|g\|_{0}\right) t\left\|\boldsymbol{\tau}_{h}\right\|_{0} .
$$

Collecting (3.50), (3.52), (3.61) and (3.62), from (3.47) we get

$$
\begin{aligned}
& \mathcal{A}\left(\boldsymbol{\theta}_{h}-\boldsymbol{\theta}_{I}, w_{h}^{*}-w_{I}^{*}, \gamma_{h}-\gamma_{I} ; \boldsymbol{\eta}_{h}, v_{h}^{*}, \boldsymbol{\tau}_{h}\right) \\
& \leq C h\left(\|g\|_{-1}+t\|g\|_{0}\right)\left(\left\|\left(\boldsymbol{\eta}_{h}, v_{h}^{*}\right)\right\|_{h}+\left\|\boldsymbol{\tau}_{h}\right\|_{-1}+t\left\|\boldsymbol{\tau}_{h}\right\|_{0}\right) .
\end{aligned}
$$


Estimate (3.43) now follows from (3.45), (3.46), (3.63) and the triangle inequality.

Using the technique in [10], one may also get the following improved estimates.

Proposition 3.4. Suppose that $\Omega$ is a convex polygon and $g \in L^{2}(\Omega)$. Then the following a priori estimates holds

$$
\begin{gathered}
\left\|\boldsymbol{\theta}-\boldsymbol{\theta}_{h}\right\|_{0} \leq C h^{2}\left(\|g\|_{-1}+t\|g\|_{0}\right) \\
\left\|w-w_{h}^{*}\right\|_{1} \leq C h(h+t)\left(\|g\|_{-1}+t\|g\|_{0}\right) .
\end{gathered}
$$

4. A posteriori error estimates. The aim of this section is to introduce suitable error estimator for the elements based on the 'Linked Interpolation Technique', and to prove its reliability and efficiency. To begin, for each $T \in \mathcal{T}_{h}$ and $e \in \mathcal{E}_{h}$ we introduce the following quantities

$$
\begin{aligned}
& \widetilde{\eta}_{T}^{2}:=h_{T}^{2} \| \operatorname{div} \mathbf{C} \varepsilon\left(\boldsymbol{\theta}_{h}\right)+\gamma_{h}\left\|_{0, T}^{2}+h_{T}^{2}\left(h_{T}^{2}+t^{2}\right)\right\| \operatorname{div} \gamma_{h}+g_{h} \|_{0, T}^{2} \\
&+\frac{1}{h_{T}^{2}+t^{2}}\left\|\mu^{-1} t^{2} \gamma_{h}-\left(\boldsymbol{\nabla} w_{h}^{*}-\boldsymbol{\theta}_{h}\right)\right\|_{0, T}^{2}, \\
& \eta_{e}^{2}:=h_{e}\left\|\llbracket \mathbf{C} \varepsilon\left(\boldsymbol{\theta}_{h}\right) \mathbf{n} \rrbracket\right\|_{0, e}^{2}+h_{e}\left(h_{e}^{2}+t^{2}\right)\left\|\llbracket \gamma_{h} \cdot \mathbf{n} \rrbracket\right\|_{0, e}^{2},
\end{aligned}
$$

where $g_{h}$ is some approximation of the load $g$. Moreover, $h_{e}$ is the length of the side $e$ and $\llbracket \cdot \rrbracket$ denotes the jump operator. We then define a local indicator $\eta_{T}$ as

$$
\eta_{T}:=\left(\widetilde{\eta}_{T}^{2}+\sum_{e \subset \partial T} \eta_{e}^{2}\right)^{1 / 2},
$$

and a global indicator $\eta$ as

$$
\eta:=\left(\sum_{T \in \mathcal{T}_{h}} \widetilde{\eta}_{T}^{2}+\sum_{e \in \mathcal{E}_{h}} \eta_{e}^{2}\right)^{1 / 2} .
$$

REMARK 4.1. When considering the element described in Section 3.1.1, the expression in (4.1) becomes simpler, since we locally have $\operatorname{div} \gamma_{h}=0$ (see (3.9)).

We now introduce some useful notation: given a generic $e \in \mathcal{E}_{h}$, we denote with $\omega_{e}$ the union of the triangles in $\mathcal{T}_{h}$ having $e$ as a side. Furthermore, for $T \in \mathcal{T}_{h}$ we set $\omega_{T}$ as the union of the $\omega_{e}$ 's, with $e \subset \partial T$. We proceed with the following result.

Lemma 4.1. Given $e \in \mathcal{E}_{h}$, let $P_{k}(e)$ be the space of polynomials of degree at most $k$ defined on $e$. There exists a linear operator

$$
\Pi_{e}: P_{k}(e) \longrightarrow H_{0}^{2}\left(\omega_{e}\right)
$$

such that for all $p_{k} \in P_{k}(e)$ it holds

$$
\begin{aligned}
& C_{1}\left\|p_{k}\right\|_{0, \omega_{e}}^{2} \leq \int_{e} p_{k}\left(\Pi_{e} p_{k}\right) \leq\left\|p_{k}\right\|_{0, \omega_{e}}^{2} \\
& \left\|\Pi_{e} p_{k}\right\|_{0, \omega_{e}} \leq C_{2} h_{e}^{1 / 2}\left\|p_{k}\right\|_{0, e} \\
& \left|\nabla\left(\Pi_{e} p_{k}\right)\right|_{0, \omega_{e}} \leq C_{3} h_{e}^{-1 / 2}\left\|p_{k}\right\|_{0, e} \\
& \left|\nabla\left(\Pi_{e} p_{k}\right)\right|_{1, \omega_{e}} \leq C_{4} h_{e}^{-3 / 2}\left\|p_{k}\right\|_{0, e} .
\end{aligned}
$$

Above, the constants $C_{i}$ depend only on $k$ and on the minimum angle of the triangles in the meshes $\mathcal{T}_{h}$. 


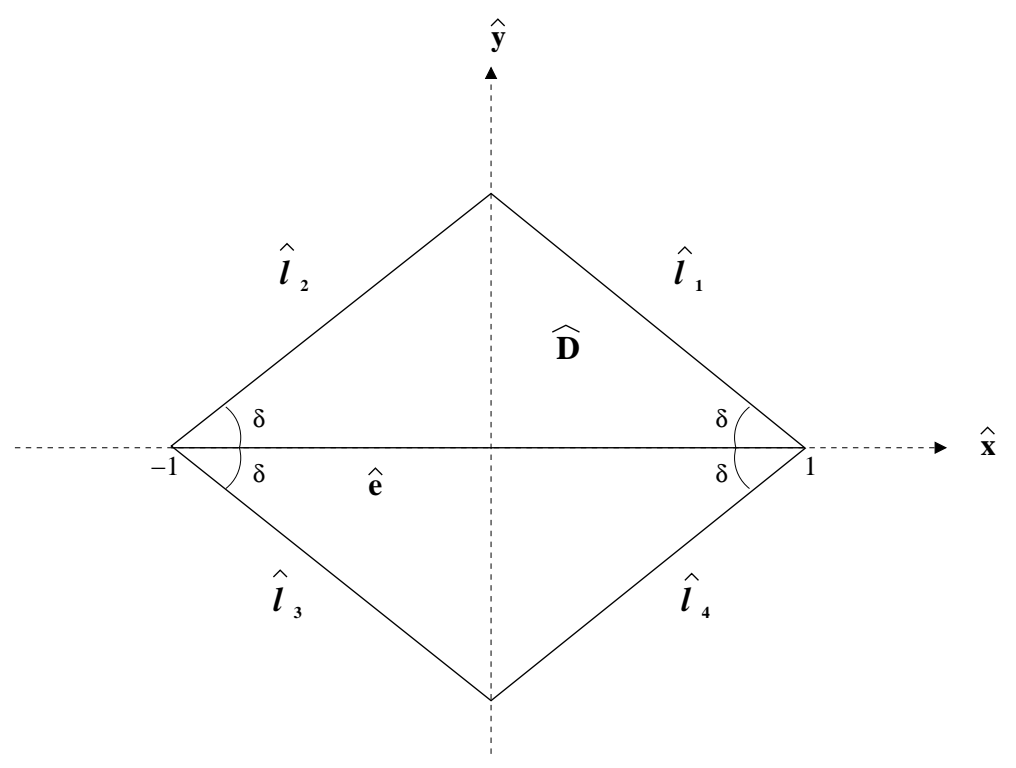

FIG. 4.1. The 'reference' rhomb $\widehat{D}$

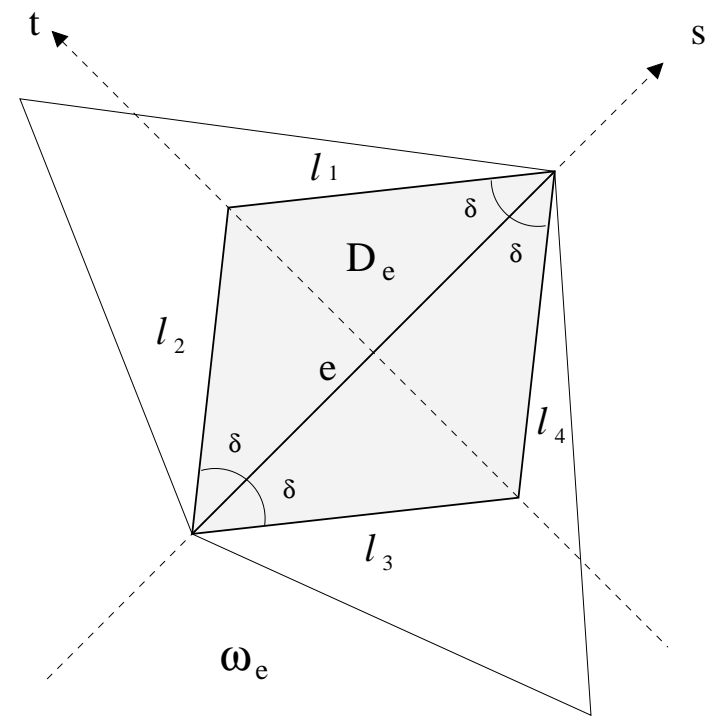

FIG. 4.2. Relevant objects associated with the edge $e$

Proof. We consider only the case of an interior edge $e$ : if $e$ is a boundary edge (i.e. $e \subset \partial \Omega$ ), the required modifications are obvious. Due to the minimum angle condition, there exists a fixed 'reference' rhomb $\widehat{D}$, as depicted in Fig. 4.1, where e.g. $\delta=\alpha / 2$ (see (3.2)), and with the following property: for each $e \in \mathcal{E}_{h}$ it is possible to determine a rhomb $D_{e} \subseteq \omega_{e}$ similar to $\widehat{D}$ (see Fig. 4.2). According to Fig. 4.2, on $\omega_{e}$ we now introduce local Cartesian coordinates $(s, t)$, as well as the functions

$$
d_{i}(s, t)=\text { "distance of }(s, t) \text { from the edge } l_{i} ", i=1, \ldots, 4 \text { (see Fig. 4.2). }
$$

Next, we define $\psi_{e}(s, t): \omega_{e} \longrightarrow \mathbf{R}$ as

$$
\psi_{e}(s, t):=\alpha_{e} \chi_{D_{e}}(s, t) \prod_{i=1}^{4} d_{i}(s, t)^{2},
$$

where $\chi_{D_{e}}(s, t)$ is the characteristic function of the set $D_{e}$, while $\alpha_{e}$ is a normalization constant in order to have $\left\|\psi_{e}\right\|_{\infty}=1$. We also notice that in the coordinates $(s, t)$ a 
generic polynomial $p_{k} \in P_{k}(e)$ can be simply written as $p_{k}(s)$. We are ready to define $\Pi_{e}: P_{k}(e) \longrightarrow H_{0}^{2}\left(\omega_{e}\right)$ by setting

$$
\left(\Pi_{e} p_{k}\right)(s, t):=\psi_{e}(s, t) p_{k}(s) \quad(s, t) \in \omega_{e} .
$$

Estimates (4.6)-(4.9) easily follows from standard scaling arguments, using the fixed reference rhomb $\widehat{D}$.

4.1. Upper bounds. We now prove that the indicator just introduced can be used as a reliable error estimator. We need to make the following

Saturation assumption: Given a mesh $\mathcal{T}_{h}$, let $\mathcal{T}_{h / 2}$ be the mesh obtained from $\mathcal{T}_{h}$ splitting each $T \in \mathcal{T}_{h}$ into four triangles using the edge midpoints. Let $\left(\boldsymbol{\theta}_{h / 2}, w_{h / 2}^{*}, \boldsymbol{\gamma}_{h / 2}\right)$ be the discrete solution corresponding to the mesh $\mathcal{T}_{h / 2}$. We assume that there exists $0<\rho<1$ such that

$$
\begin{aligned}
\|\left(\boldsymbol{\theta}-\boldsymbol{\theta}_{h / 2}, w\right. & \left.-w_{h / 2}^{*}\right)\left\|_{h / 2}+\right\| \boldsymbol{\gamma}-\gamma_{h / 2}\left\|_{-1}+t\right\| \boldsymbol{\gamma}-\gamma_{h / 2} \|_{0} \\
& \leq \rho\left(\left\|\left(\boldsymbol{\theta}-\boldsymbol{\theta}_{h}, w-w_{h}^{*}\right)\right\|_{h}+\left\|\boldsymbol{\gamma}-\gamma_{h}\right\|_{-1}+t\left\|\boldsymbol{\gamma}-\gamma_{h}\right\|_{0}\right) .
\end{aligned}
$$

By using the saturation assumption (4.13), it is easily seen that one gets the reliability estimate

$$
\begin{aligned}
\left\|\left(\boldsymbol{\theta}-\boldsymbol{\theta}_{h}, w-w_{h}^{*}\right)\right\|_{h} & +\left\|\boldsymbol{\gamma}-\gamma_{h}\right\|_{-1}+t\left\|\boldsymbol{\gamma}-\gamma_{h}\right\|_{0} \\
& \leq C\left(\sum_{T \in \mathcal{T}_{h}}\left(\eta_{T}^{2}+h_{T}^{2}\left(h_{T}^{2}+t^{2}\right)\left\|g-g_{h}\right\|_{0, T}^{2}\right)\right)^{1 / 2},
\end{aligned}
$$

provided one is able to bound

$$
\left\|\left(\boldsymbol{\theta}_{h / 2}-\boldsymbol{\theta}_{h}, w_{h / 2}^{*}-w_{h}^{*}\right)\right\|_{h / 2}+\left\|\gamma_{h / 2}-\gamma_{h}\right\|_{-1}+t\left\|\gamma_{h / 2}-\gamma_{h}\right\|_{0} .
$$

To this aim, we need the next result, which states that functions in $\boldsymbol{X}_{h / 2}$ can be approximated by functions in $\boldsymbol{X}_{h}$. The proof can be performed by scaling arguments, using exactly the same techniques of Lemma 3.1 in [4], and recalling the norm definition (3.21).

Lemma 4.2. Given $\left(\boldsymbol{\eta}_{h / 2}, v_{h / 2}^{*}\right) \in \boldsymbol{X}_{h / 2}$, there exists $\left(\boldsymbol{\eta}_{h}, v_{h}^{*}\right) \in \boldsymbol{X}_{h}$ such that

$$
\begin{aligned}
& \sum_{T \in \mathcal{T}_{h}} h_{T}^{-2}\left(\left\|\boldsymbol{\eta}_{h / 2}-\boldsymbol{\eta}_{h}\right\|_{0, T}^{2}+\frac{1}{h_{T}^{2}+t^{2}}\left\|v_{h / 2}^{*}-v_{h}^{*}\right\|_{0, T}^{2}\right) \\
& \quad+\sum_{e \in \mathcal{E}_{h}} h_{e}^{-1}\left(\left\|\boldsymbol{\eta}_{h / 2}-\boldsymbol{\eta}_{h}\right\|_{0, e}^{2}+\frac{1}{h_{e}^{2}+t^{2}}\left\|v_{h / 2}^{*}-v_{h}^{*}\right\|_{0, e}^{2}\right) \leq C\left\|\left(\boldsymbol{\eta}_{h / 2}, v_{h / 2}^{*}\right)\right\|_{h / 2}^{2} .
\end{aligned}
$$

We are now ready to prove the following proposition.

Proposition 4.3. We have

$$
\begin{aligned}
\left\|\left(\boldsymbol{\theta}_{h / 2}-\boldsymbol{\theta}_{h}, w_{h / 2}^{*}-w_{h}^{*}\right)\right\|_{h / 2}+\left\|\gamma_{h / 2}-\gamma_{h}\right\|_{-1}+t\left\|\gamma_{h / 2}-\gamma_{h}\right\|_{0} \\
\leq C\left(\sum_{T \in \mathcal{T}_{h}}\left(\eta_{T}^{2}+h_{T}^{2}\left(h_{T}^{2}+t^{2}\right)\left\|g-g_{h}\right\|_{0, T}^{2}\right)\right)^{1 / 2} .
\end{aligned}
$$

Proof. Consider $\left(\boldsymbol{\theta}_{h / 2}-\boldsymbol{\theta}_{h}, w_{h / 2}^{*}-w_{h}^{*} ; \boldsymbol{\gamma}_{h / 2}-\boldsymbol{\gamma}_{h}\right) \in \boldsymbol{X}_{h / 2} \times \boldsymbol{\Gamma}_{h / 2}$. Discrete stability for the $\mathcal{T}_{h / 2}$-problem (see Proposition 3.2) implies that there exists $\left(\boldsymbol{\eta}_{h / 2}, v_{h / 2}^{*} ; \boldsymbol{\tau}_{h / 2}\right)$ in $\boldsymbol{X}_{h / 2} \times \boldsymbol{\Gamma}_{h / 2}$ such that 


$$
\left\|\left(\boldsymbol{\eta}_{h / 2}, v_{h / 2}^{*}\right)\right\|_{h / 2}+\left\|\boldsymbol{\tau}_{h / 2}\right\|_{-1}+t\left\|\boldsymbol{\tau}_{h / 2}\right\|_{0} \leq 1
$$

and

$$
\begin{aligned}
& C\left(\left\|\left(\boldsymbol{\theta}_{h / 2}-\boldsymbol{\theta}_{h}, w_{h / 2}^{*}-w_{h}^{*}\right)\right\|_{h / 2}+\left\|\boldsymbol{\gamma}_{h / 2}-\gamma_{h}\right\|_{-1}+t\left\|\gamma_{h / 2}-\gamma_{h}\right\|_{0}\right) \\
& \leq\left\{a\left(\boldsymbol{\theta}_{h / 2}-\boldsymbol{\theta}_{h}, \boldsymbol{\eta}_{h / 2}\right)+\left(\gamma_{h / 2}-\boldsymbol{\gamma}_{h}, \boldsymbol{\nabla} v_{h / 2}^{*}-\boldsymbol{\eta}_{h / 2}\right)\right\} \\
&+\left\{-\left(\boldsymbol{\nabla}\left(w_{h / 2}^{*}-w_{h}^{*}\right)-\left(\boldsymbol{\theta}_{h / 2}-\boldsymbol{\theta}_{h}\right), \boldsymbol{\tau}_{h / 2}\right)+\mu^{-1} t^{2}\left(\boldsymbol{\gamma}_{h / 2}-\boldsymbol{\gamma}_{h}, \boldsymbol{\tau}_{h / 2}\right)\right\} \\
&=(I)+(I I) .
\end{aligned}
$$

On one hand, since $\left(\boldsymbol{\theta}_{h / 2}, w_{h / 2}^{*} ; \gamma_{h / 2}\right)\left(\right.$ resp. $\left.\left(\boldsymbol{\theta}_{h}, w_{h}^{*} ; \boldsymbol{\gamma}_{h}\right)\right)$ solves the discrete problem with respect to the mesh $\mathcal{T}_{h / 2}\left(\right.$ resp. $\left.\mathcal{T}_{h}\right)$, we have

$$
\begin{aligned}
(I) & =a\left(\boldsymbol{\theta}_{h / 2}-\boldsymbol{\theta}_{h}, \boldsymbol{\eta}_{h / 2}\right)+\left(\boldsymbol{\gamma}_{h / 2}-\boldsymbol{\gamma}_{h}, \boldsymbol{\nabla} v_{h / 2}^{*}-\boldsymbol{\eta}_{h / 2}\right) \\
& =\left(g, v_{h / 2}^{*}\right)-a\left(\boldsymbol{\theta}_{h}, \boldsymbol{\eta}_{h / 2}\right)-\left(\boldsymbol{\gamma}_{h}, \boldsymbol{\nabla} v_{h / 2}^{*}-\boldsymbol{\eta}_{h / 2}\right) \\
& =\left(g, v_{h / 2}^{*}-v_{h}^{*}\right)-a\left(\boldsymbol{\theta}_{h}, \boldsymbol{\eta}_{h / 2}-\boldsymbol{\eta}_{h}\right)-\left(\boldsymbol{\gamma}_{h}, \boldsymbol{\nabla}\left(v_{h / 2}^{*}-v_{h}^{*}\right)-\left(\boldsymbol{\eta}_{h / 2}-\boldsymbol{\eta}_{h}\right)\right),
\end{aligned}
$$

where we choose $\left(\boldsymbol{\eta}_{h}, v_{h}^{*}\right) \in \boldsymbol{X}_{h}$ satisfying estimate (4.16). An elementwise integration by parts gives

$$
\begin{aligned}
(I) & =\sum_{T \in \mathcal{T}_{h}}\left\{\int_{T}\left(\operatorname{div} \mathbf{C} \varepsilon\left(\boldsymbol{\theta}_{h}\right)+\boldsymbol{\gamma}_{h}\right) \cdot\left(\boldsymbol{\eta}_{h / 2}-\boldsymbol{\eta}_{h}\right)-\int_{\partial T} \mathbf{C} \varepsilon\left(\boldsymbol{\theta}_{h}\right) \mathbf{n} \cdot\left(\boldsymbol{\eta}_{h / 2}-\boldsymbol{\eta}_{h}\right)\right\} \\
& +\sum_{T \in \mathcal{T}_{h}}\left\{\int_{T}\left(\operatorname{div} \gamma_{h}+g\right)\left(v_{h / 2}^{*}-v_{h}^{*}\right)-\int_{\partial T} \boldsymbol{\gamma}_{h} \cdot \mathbf{n}\left(v_{h / 2}^{*}-v_{h}^{*}\right)\right\}
\end{aligned}
$$

by which

$$
\begin{aligned}
(I) & =\sum_{T \in \mathcal{T}_{h}} \int_{T}\left(\operatorname{div} \mathbf{C} \varepsilon\left(\boldsymbol{\theta}_{h}\right)+\boldsymbol{\gamma}_{h}\right) \cdot\left(\boldsymbol{\eta}_{h / 2}-\boldsymbol{\eta}_{h}\right)-\sum_{e \in \mathcal{E}_{h}} \int_{e} \llbracket \mathbf{C} \varepsilon\left(\boldsymbol{\theta}_{h}\right) \mathbf{n} \rrbracket \cdot\left(\boldsymbol{\eta}_{h / 2}-\boldsymbol{\eta}_{h}\right) \\
& +\sum_{T \in \mathcal{T}_{h}} \int_{T}\left(\operatorname{div} \gamma_{h}+g\right)\left(v_{h / 2}^{*}-v_{h}^{*}\right)-\sum_{e \in \mathcal{E}_{h}} \int_{e} \llbracket \gamma_{h} \cdot \mathbf{n} \rrbracket\left(v_{h / 2}^{*}-v_{h}^{*}\right) .
\end{aligned}
$$

Hence, it holds

$$
\begin{aligned}
& (I) \leq C\left(\left(\sum_{T \in \mathcal{T}_{h}} h_{T}^{2}\left\|\operatorname{div} \mathbf{C} \varepsilon\left(\boldsymbol{\theta}_{h}\right)+\gamma_{h}\right\|_{0, T}^{2}\right)^{1 / 2}\left(\sum_{T \in \mathcal{T}_{h}} h_{T}^{-2}\left\|\boldsymbol{\eta}_{h / 2}-\boldsymbol{\eta}_{h}\right\|_{0, T}^{2}\right)^{1 / 2}\right. \\
& +\left(\sum_{e \in \mathcal{E}_{h}} h_{e}\left\|\llbracket \mathbf{C} \varepsilon\left(\boldsymbol{\theta}_{h}\right) \mathbf{n} \rrbracket\right\|_{0, e}^{2}\right)^{1 / 2}\left(\sum_{e \in \mathcal{E}_{h}} h_{e}^{-1}\left\|\boldsymbol{\eta}_{h / 2}-\boldsymbol{\eta}_{h}\right\|_{0, e}^{2}\right)^{1 / 2} \\
& +\left(\sum_{T \in \mathcal{T}_{h}} h_{T}^{2}\left(h_{T}^{2}+t^{2}\right)\left\|\operatorname{div} \gamma_{h}+g\right\|_{0, T}^{2}\right)^{1 / 2}\left(\sum_{T \in \mathcal{T}_{h}} \frac{1}{h_{T}^{2}\left(h_{T}^{2}+t^{2}\right)}\left\|v_{h / 2}^{*}-v_{h}^{*}\right\|_{0, T}^{2}\right)^{1 / 2} \\
& \left.+\left(\sum_{e \in \mathcal{E}_{h}} h_{e}\left(h_{e}^{2}+t^{2}\right)\left\|\llbracket \gamma_{h} \cdot \mathbf{n} \rrbracket\right\|_{0, e}^{2}\right)^{1 / 2}\left(\sum_{e \in \mathcal{E}_{h}} \frac{1}{h_{e}\left(h_{e}^{2}+t^{2}\right)}\left\|v_{h / 2}^{*}-v_{h}^{*}\right\|_{0, e}^{2}\right)^{1 / 2}\right) .
\end{aligned}
$$


Using Lemma 4.2, we get

$$
\begin{aligned}
& (I) \leq C\left(\left(\sum_{T \in \mathcal{T}_{h}} h_{T}^{2}\left\|\operatorname{div} \mathbf{C} \varepsilon\left(\boldsymbol{\theta}_{h}\right)+\boldsymbol{\gamma}_{h}\right\|_{0, T}^{2}\right)^{1 / 2}+\left(\sum_{e \in \mathcal{E}_{h}} h_{e}\left\|\llbracket \mathbf{C} \varepsilon\left(\boldsymbol{\theta}_{h}\right) \mathbf{n} \rrbracket\right\|_{0, e}^{2}\right)^{1 / 2}\right. \\
& \left.+\left(\sum_{T \in \mathcal{T}_{h}} h_{T}^{2}\left(h_{T}^{2}+t^{2}\right)\left\|\operatorname{div} \boldsymbol{\gamma}_{h}+g\right\|_{0, T}^{2}\right)^{1 / 2}+\left(\sum_{e \in \mathcal{E}_{h}} h_{e}\left(h_{e}^{2}+t^{2}\right)\left\|\llbracket \boldsymbol{\gamma}_{h} \cdot \mathbf{n} \rrbracket\right\|_{0, e}^{2}\right)^{1 / 2}\right) \\
& \times\left\|\left(\boldsymbol{\eta}_{h / 2}, v_{h / 2}^{*}\right)\right\|_{h / 2} .
\end{aligned}
$$

Therefore, one has

$$
\begin{aligned}
& (I) \leq C\left(\left(\sum_{T \in \mathcal{T}_{h}} h_{T}^{2}\left\|\operatorname{div} \mathbf{C} \varepsilon\left(\boldsymbol{\theta}_{h}\right)+\gamma_{h}\right\|_{0, T}^{2}\right)^{1 / 2}+\left(\sum_{e \in \mathcal{E}_{h}} h_{e}\left\|\llbracket \mathbf{C} \varepsilon\left(\boldsymbol{\theta}_{h}\right) \mathbf{n} \rrbracket\right\|_{0, e}^{2}\right)^{1 / 2}\right. \\
& +\left(\sum_{T \in \mathcal{T}_{h}} h_{T}^{2}\left(h_{T}^{2}+t^{2}\right)\left\|\operatorname{div} \gamma_{h}+g_{h}\right\|_{0, T}^{2}\right)^{1 / 2}+\left(\sum_{T \in \mathcal{T}_{h}} h_{T}^{2}\left(h_{T}^{2}+t^{2}\right)\left\|g-g_{h}\right\|_{0, T}^{2}\right)^{1 / 2} \\
& \left.+\left(\sum_{e \in \mathcal{E}_{h}} h_{e}\left(h_{e}^{2}+t^{2}\right)\left\|\llbracket \gamma_{h} \cdot \mathbf{n} \rrbracket\right\|_{0, e}^{2}\right)^{1 / 2}\right)\left\|\left(\boldsymbol{\eta}_{h / 2}, v_{h / 2}^{*}\right)\right\|_{h / 2} .
\end{aligned}
$$

On the other hand, since $\left(\boldsymbol{\theta}_{h / 2}, w_{h / 2}^{*} ; \gamma_{h / 2}\right)$ solves the discrete problem with respect to the mesh $\mathcal{T}_{h / 2}$, we have

$$
\begin{aligned}
& (I I)=-\left(\boldsymbol{\nabla}\left(w_{h / 2}^{*}-w_{h}^{*}\right)-\left(\boldsymbol{\theta}_{h / 2}-\boldsymbol{\theta}_{h}\right), \boldsymbol{\tau}_{h / 2}\right)+\mu^{-1} t^{2}\left(\boldsymbol{\gamma}_{h / 2}-\boldsymbol{\gamma}_{h}, \boldsymbol{\tau}_{h / 2}\right) \\
& =-\left(\mu^{-1} t^{2} \boldsymbol{\gamma}_{h}-\left(\boldsymbol{\nabla} w_{h}^{*}-\boldsymbol{\theta}_{h}\right), \boldsymbol{\tau}_{h / 2}\right) \\
& \leq\left(\sum_{T \in \mathcal{T}_{h}} \frac{1}{h_{T}^{2}+t^{2}}\left\|\mu^{-1} t^{2} \boldsymbol{\gamma}_{h}-\left(\boldsymbol{\nabla} w_{h}^{*}-\boldsymbol{\theta}_{h}\right)\right\|_{0, T}^{2}\right)^{1 / 2}\left(\sum_{T \in \mathcal{T}_{h}}\left(h_{T}^{2}+t^{2}\right)\left\|\boldsymbol{\tau}_{h / 2}\right\|_{0, T}^{2}\right)^{1 / 2} \\
& \leq C\left(\sum_{T \in \mathcal{T}_{h}} \frac{1}{h_{T}^{2}+t^{2}}\left\|\mu^{-1} t^{2} \boldsymbol{\gamma}_{h}-\left(\boldsymbol{\nabla} w_{h}^{*}-\boldsymbol{\theta}_{h}\right)\right\|_{0, T}^{2}\right)^{1 / 2}\left(\left\|\boldsymbol{\tau}_{h / 2}\right\|_{-1}+t\left\|\boldsymbol{\tau}_{h / 2}\right\|_{0}\right)
\end{aligned}
$$

As a consequence, from (4.19), (4.25), (4.26), using (4.18) and recalling definitions (4.1)-(4.3), we have

$$
\begin{gathered}
\left\|\left(\boldsymbol{\theta}_{h / 2}-\boldsymbol{\theta}_{h}, w_{h / 2}^{*}-w_{h}^{*}\right)\right\|_{h / 2}+\left\|\gamma_{h / 2}-\gamma_{h}\right\|_{-1}+t\left\|\gamma_{h / 2}-\gamma_{h}\right\|_{0} \\
\leq C\left(\sum_{T \in \mathcal{T}_{h}}\left(\eta_{T}^{2}+h_{T}^{2}\left(h_{T}^{2}+t^{2}\right)\left\|g-g_{h}\right\|_{0, T}^{2}\right)\right)^{1 / 2}
\end{gathered}
$$

The proof is complete.

4.2. Lower bounds. We now prove the efficiency of our error estimator by establishing the following proposition. 
Proposition 4.4. Let $(\boldsymbol{\theta}, w ; \boldsymbol{\gamma})$ (resp. $\left.\left(\boldsymbol{\theta}_{h}, w_{h}^{*} ; \boldsymbol{\gamma}_{h}\right)\right)$ be the solution of the continuous (resp. discrete) problem. Given $T \in \mathcal{T}_{h}$, it holds

$$
\begin{aligned}
\eta_{T} \leq C & \left(\frac{1}{\left(h_{T}^{2}+t^{2}\right)^{1 / 2}}\left\|\boldsymbol{\nabla}\left(w_{h}^{*}-w\right)-\left(\boldsymbol{\theta}_{h}-\boldsymbol{\theta}\right)\right\|_{0, T}+\left\|\boldsymbol{\theta}_{h}-\boldsymbol{\theta}\right\|_{1, \omega_{T}}\right. \\
& \left.+\left.\left\|\gamma_{h}-\gamma\right\|\right|_{-1, \omega_{T}}+t\left\|\gamma_{h}-\gamma\right\|_{0, \omega_{T}}+\left(\sum_{T \subset \omega_{T}} h_{T}^{2}\left(h_{T}^{2}+t^{2}\right)\left\|g-g_{h}\right\|_{0, T}^{2}\right)^{1 / 2}\right)
\end{aligned}
$$

where $\eta_{T}$ is defined by (4.1)-(4.3).

Proof. Fix $T \in \mathcal{T}_{h}$ and a generic edge $e \subset \partial T$. We proceed in three steps. First step. Since

$$
\mu^{-1} t^{2} \gamma=\nabla w-\boldsymbol{\theta}
$$

we get

$$
\begin{aligned}
& \frac{1}{\left(h_{T}^{2}+t^{2}\right)^{1 / 2}}\left\|\mu^{-1} t^{2} \gamma_{h}-\left(\nabla w_{h}^{*}-\boldsymbol{\theta}_{h}\right)\right\|_{0, T} \\
& =\frac{1}{\left(h_{T}^{2}+t^{2}\right)^{1 / 2}}\left\|\mu^{-1} t^{2}\left(\gamma_{h}-\gamma\right)-\left(\nabla\left(w_{h}^{*}-w\right)-\left(\boldsymbol{\theta}_{h}-\boldsymbol{\theta}\right)\right)\right\| \|_{0, T} \\
& \leq C\left(t\left\|\gamma_{h}-\gamma\right\|_{0, T}+\frac{1}{\left(h_{T}^{2}+t^{2}\right)^{1 / 2}}\left\|\nabla\left(w_{h}^{*}-w\right)-\left(\boldsymbol{\theta}_{h}-\boldsymbol{\theta}\right)\right\|_{0, T}\right)
\end{aligned}
$$

Second step. We choose

$$
\boldsymbol{\eta}_{T}=h_{T}^{2}\left(\operatorname{div} \mathbf{C} \varepsilon\left(\boldsymbol{\theta}_{h}\right)+\boldsymbol{\gamma}_{h}\right) b_{T}
$$

where $b_{T}$ is the standard cubic bubble on $T$. We observe that

$$
\left|\boldsymbol{\eta}_{T}\right|_{1, T} \leq C h_{T}|| \operatorname{div} \mathbf{C} \varepsilon\left(\boldsymbol{\theta}_{h}\right)+\gamma_{h} \|_{0, T} .
$$

Taking advantage of the equilibrium equation

$$
-\operatorname{div} \mathbf{C} \varepsilon(\boldsymbol{\theta})-\boldsymbol{\gamma}=\mathbf{0}
$$

we get

$$
\begin{aligned}
h_{T}^{2} \| & \operatorname{div} \mathbf{C} \varepsilon\left(\boldsymbol{\theta}_{h}\right)+\boldsymbol{\gamma}_{h} \|_{0, T}^{2} \\
& \leq C\left(\operatorname{div} \mathbf{C} \varepsilon\left(\boldsymbol{\theta}_{h}\right)+\boldsymbol{\gamma}_{h}, \boldsymbol{\eta}_{T}\right)=C\left(\operatorname{div} \mathbf{C} \varepsilon\left(\boldsymbol{\theta}_{h}-\boldsymbol{\theta}\right)+\left(\boldsymbol{\gamma}_{h}-\boldsymbol{\gamma}\right), \boldsymbol{\eta}_{T}\right) \\
& =C\left(-a\left(\boldsymbol{\theta}_{h}-\boldsymbol{\theta}, \boldsymbol{\eta}_{T}\right)+\left(\boldsymbol{\gamma}_{h}-\boldsymbol{\gamma}, \boldsymbol{\eta}_{T}\right)\right) \\
& \leq C\left(\left\|\boldsymbol{\theta}_{h}-\boldsymbol{\theta}\right\|_{1, T}+\left\|\boldsymbol{\gamma}_{h}-\boldsymbol{\gamma}\right\|_{-1, T}\right)\left|\boldsymbol{\eta}_{T}\right|_{1, T} .
\end{aligned}
$$

Using (4.32), from (4.34) we thus obtain

$$
h_{T}\left\|\operatorname{div} \mathbf{C} \varepsilon\left(\boldsymbol{\theta}_{h}\right)+\boldsymbol{\gamma}_{h}\right\|_{0, T} \leq C\left(\left\|\boldsymbol{\theta}_{h}-\boldsymbol{\theta}\right\|_{1, T}+\left\|\boldsymbol{\gamma}_{h}-\boldsymbol{\gamma}\right\|_{-1, T}\right) .
$$

Next, we choose

$$
\boldsymbol{\eta}_{e}=h_{e} P\left(\llbracket \mathbf{C} \varepsilon\left(\boldsymbol{\theta}_{h}\right) \mathbf{n} \rrbracket\right) b_{e},
$$

where $P$ is the prolongation operator introduced in [25] and $b_{e}$ is the usual 'edge' bubble on $e$. We observe that it holds

$$
\left(\sum_{T \subset \omega_{e}} h_{T}^{-2}\left\|\boldsymbol{\eta}_{e}\right\|_{0, T}^{2}\right)^{1 / 2} \leq C\left|\boldsymbol{\eta}_{e}\right|_{1, \omega_{e}} \leq C h_{e}^{1 / 2}\left\|\llbracket \mathbf{C} \varepsilon\left(\boldsymbol{\theta}_{h}\right) \mathbf{n} \rrbracket\right\|_{0, e} .
$$


Integrating by parts and using again the equilibrium equation (4.33), we have

$$
\begin{aligned}
h_{e} \| & \llbracket \mathbf{C} \varepsilon\left(\boldsymbol{\theta}_{h}\right) \mathbf{n} \rrbracket \|_{0, e}^{2} \\
& \leq C \int_{e} \llbracket \mathbf{C} \varepsilon\left(\boldsymbol{\theta}_{h}\right) \mathbf{n} \rrbracket \cdot \boldsymbol{\eta}_{e}=C\left(\int_{\omega_{e}} \operatorname{div} \mathbf{C} \varepsilon\left(\boldsymbol{\theta}_{h}\right) \cdot \boldsymbol{\eta}_{e}+\int_{\omega_{e}} \mathbf{C} \varepsilon\left(\boldsymbol{\theta}_{h}\right): \varepsilon\left(\boldsymbol{\eta}_{e}\right)\right) \\
& =C\left(\left(\operatorname{div} \mathbf{C} \varepsilon\left(\boldsymbol{\theta}_{h}\right)+\boldsymbol{\gamma}_{h}, \boldsymbol{\eta}_{e}\right)+a\left(\boldsymbol{\theta}_{h}-\boldsymbol{\theta}, \boldsymbol{\eta}_{e}\right)-\left(\boldsymbol{\gamma}_{h}-\boldsymbol{\gamma}, \boldsymbol{\eta}_{e}\right)\right) \\
& \leq C\left(\left(\sum_{T \subset \omega_{e}} h_{T}^{2}\left\|\operatorname{div} \mathbf{C} \varepsilon\left(\boldsymbol{\theta}_{h}\right)+\boldsymbol{\gamma}_{h}\right\|_{0, T}^{2}\right)^{1 / 2}\left(\sum_{T \subset \omega_{e}} h_{T}^{-2}\left\|\boldsymbol{\eta}_{e}\right\|_{0, T}^{2}\right)^{1 / 2}\right. \\
& \left.+\left(\left\|\boldsymbol{\theta}_{h}-\boldsymbol{\theta}\right\|_{1, \omega_{e}}+\left\|\boldsymbol{\gamma}_{h}-\gamma\right\|_{-1, \omega_{e}}\right)\left|\boldsymbol{\eta}_{e}\right|_{1, \omega_{e}}\right) .
\end{aligned}
$$

Therefore, using (4.37) and (4.35), from (4.38) we get

$$
h_{e}^{1 / 2}\left\|\llbracket \mathbf{C} \varepsilon\left(\boldsymbol{\theta}_{h}\right) \mathbf{n} \rrbracket\right\|_{0, e} \leq C\left(\left\|\boldsymbol{\theta}_{h}-\boldsymbol{\theta}\right\|_{1, \omega_{e}}+\left\|\boldsymbol{\gamma}_{h}-\gamma\right\|_{-1, \omega_{e}}\right) .
$$

Third step. We first define

$$
\varphi_{T}=\left(\operatorname{div} \gamma_{h}+g_{h}\right) b_{T}^{2} .
$$

We observe that $\varphi_{T} \in H_{0}^{2}(T)$ and one has

$$
\begin{aligned}
& \left|\varphi_{T}\right|_{1, T} \leq C h_{T}^{-1}\left\|\operatorname{div} \gamma_{h}+g_{h}\right\|_{0, T} \\
& \left|\nabla \varphi_{T}\right|_{1, T} \leq C h_{T}^{-2}\left\|\operatorname{div} \gamma_{h}+g_{h}\right\|_{0, T} .
\end{aligned}
$$

We then set

$$
v_{T}=h_{T}^{2}\left(h_{T}^{2}+t^{2}\right) \varphi_{T} .
$$

Using the equilibrium equation

$$
-\operatorname{div} \gamma=g
$$

we get

$$
\begin{aligned}
h_{T}^{2}\left(h_{T}^{2}+t^{2}\right) \| \operatorname{div} \gamma_{h} & +g_{h} \|_{0, T}^{2} \leq C\left(\operatorname{div} \gamma_{h}+g_{h}, v_{T}\right) \\
& =C\left(\left(\operatorname{div}\left(\gamma_{h}-\gamma\right), v_{T}\right)+\left(g_{h}-g, v_{T}\right)\right) .
\end{aligned}
$$

We now separately treat the two terms at the right-hand side of (4.44). Integrating by parts, recalling (4.40) and (4.42), and using (4.41), we have

$$
\begin{aligned}
& \left(\operatorname{div}\left(\gamma_{h}-\gamma\right), v_{T}\right)=-\left(\gamma_{h}-\gamma, \nabla v_{T}\right) \\
& =-h_{T}^{4}\left(\gamma_{h}-\gamma, \nabla \varphi_{T}\right)-t^{2} h_{T}^{2}\left(\gamma_{h}-\gamma, \nabla v_{T}\right) \\
& \leq\left\|\gamma_{h}-\gamma\right\|_{-1, T} h_{T}^{4}\left|\nabla \varphi_{T}\right|_{1, T}+t\left\|\gamma_{h}-\gamma\right\|_{0, T} h_{T}^{2} t\left\|\nabla \varphi_{T}\right\|_{0, T} \\
& \leq C\left(\left\|\gamma_{h}-\gamma\right\|_{-1, T} h_{T}^{2}\left\|\operatorname{div} \gamma_{h}+g_{h}\right\|_{0, T}+t\left\|\gamma_{h}-\gamma\right\|_{0, T} h_{T} t\left\|\operatorname{div} \gamma_{h}+g_{h}\right\|_{0, T}\right) \\
& \leq C\left(\left\|\gamma_{h}-\gamma\right\|_{-1, T}+t\left\|\gamma_{h}-\gamma\right\|_{0, T}\right) h_{T}\left(h_{T}^{2}+t^{2}\right)^{1 / 2}\left\|\operatorname{div} \gamma_{h}+g_{h}\right\|_{0, T}
\end{aligned}
$$

Furthermore, it holds

$$
\begin{aligned}
\left(g_{h}-g, v_{T}\right) & \leq h_{T}\left(h_{T}^{2}+t^{2}\right)^{1 / 2}\left\|g_{h}-g\right\|_{0, T} h_{T}\left(h_{T}^{2}+t^{2}\right)^{1 / 2}\left\|\varphi_{T}\right\|_{0, T} \\
& \leq C h_{T}\left(h_{T}^{2}+t^{2}\right)^{1 / 2}\left\|g_{h}-g\right\|_{0, T} h_{T}\left(h_{T}^{2}+t^{2}\right)^{1 / 2}\left\|\operatorname{div} \gamma_{h}+g_{h}\right\|_{0, T} .
\end{aligned}
$$


Therefore, using (4.45) and (4.46), from (4.44) we infer

$$
\begin{aligned}
h_{T}\left(h_{T}^{2}+t^{2}\right)^{1 / 2} \| & \operatorname{div} \gamma_{h}+g_{h} \|_{0, T} \leq C\left(\left\|\gamma_{h}-\gamma\right\|_{-1, T}\right. \\
& \left.+t\left\|\gamma_{h}-\gamma\right\|_{0, T}+h_{T}\left(h_{T}^{2}+t^{2}\right)^{1 / 2}\left\|g_{h}-g\right\|_{0, T}\right) .
\end{aligned}
$$

Next, we define

$$
\varphi_{e}=\Pi_{e}\left(\llbracket \gamma_{h} \cdot \mathbf{n} \rrbracket\right),
$$

where $\Pi_{e}$ is the linear operator of Lemma 4.1. Therefore, we have

$$
\begin{aligned}
& \left\|\llbracket \gamma_{h} \cdot \mathbf{n} \rrbracket\right\|_{0, \omega_{e}}^{2} \leq C \int_{e} \llbracket \gamma_{h} \cdot \mathbf{n} \rrbracket \varphi_{e} \\
& \left\|\varphi_{e}\right\|_{0, \omega_{e}} \leq C h_{e}^{1 / 2}\left\|\llbracket \gamma_{h} \cdot \mathbf{n} \rrbracket\right\|_{0, e} \\
& \left\|\nabla \varphi_{e}\right\|_{0, \omega_{e}} \leq C h_{e}^{-1 / 2}\left\|\llbracket \gamma_{h} \cdot \mathbf{n} \rrbracket\right\|_{0, e} \\
& \left|\nabla \varphi_{e}\right|_{1, \omega_{e}} \leq C h_{e}^{-3 / 2}\left\|\llbracket \gamma_{h} \cdot \mathbf{n} \rrbracket\right\|_{0, e} .
\end{aligned}
$$

We then set

$$
v_{e}=h_{e}\left(h_{e}^{2}+t^{2}\right) \varphi_{e} .
$$

Integrating by parts using (4.49) and the equilibrium equation (4.43), we get

$$
\begin{aligned}
h_{e}\left(h_{e}^{2}+t^{2}\right) \| & \llbracket \gamma_{h} \cdot \mathbf{n} \rrbracket \|_{0, e}^{2} \leq C \int_{e} \llbracket \gamma_{h} \cdot \mathbf{n} \rrbracket v_{e} \\
& \leq C\left(\int_{\omega_{e}} v_{e} \operatorname{div} \gamma_{h}+\int_{\omega_{e}} \gamma_{h} \cdot \nabla v_{e}\right) \\
& =C\left(\left(\operatorname{div} \gamma_{h}+g, v_{e}\right)+\left(\gamma_{h}-\gamma, \nabla v_{e}\right)\right) \\
& =C\left(\left(\operatorname{div} \gamma_{h}+g_{h}, v_{e}\right)+\left(g-g_{h}, v_{e}\right)+\left(\gamma_{h}-\gamma, \nabla v_{e}\right)\right) .
\end{aligned}
$$

We now estimate the three terms above. Recalling (4.53) and using (4.50), we obtain

$$
\begin{aligned}
\left(\operatorname{div} \gamma_{h}\right. & \left.+g_{h}, v_{e}\right)=h_{e}\left(h_{e}^{2}+t^{2}\right)\left(\operatorname{div} \gamma_{h}+g_{h}, \varphi_{e}\right) \\
& =\sum_{T \subset \omega_{e}} \int_{T}\left(h_{e}\left(h_{e}^{2}+t^{2}\right)^{1 / 2}\left(\operatorname{div} \gamma_{h}+g_{h}\right)\right)\left(\left(h_{e}^{2}+t^{2}\right)^{1 / 2} \varphi_{e}\right) \\
& \leq\left(\sum_{T \subset \omega_{e}} h_{e}^{2}\left(h_{e}^{2}+t^{2}\right)\left\|\operatorname{div} \gamma_{h}+g_{h}\right\|_{0, T}^{2}\right)^{1 / 2}\left(\sum_{T \subset \omega_{e}}\left(h_{e}^{2}+t^{2}\right)\left\|\varphi_{e}\right\|_{0, T}^{2}\right)^{1 / 2} \\
& \leq\left(\sum_{T \subset \omega_{e}} h_{T}^{2}\left(h_{T}^{2}+t^{2}\right)\left\|\operatorname{div} \gamma_{h}+g_{h}\right\|_{0, T}^{2}\right)^{1 / 2}\left(\sum_{T \subset \omega_{e}}\left(h_{e}^{2}+t^{2}\right)\left\|\varphi_{e}\right\|_{0, T}^{2}\right)^{1 / 2} \\
& \leq C\left(\sum_{T \subset \omega_{e}} h_{T}^{2}\left(h_{T}^{2}+t^{2}\right)\left\|\operatorname{div} \gamma_{h}+g_{h}\right\|_{0, T}^{2}\right)^{1 / 2} h_{e}^{1 / 2}\left(h_{e}^{2}+t^{2}\right)^{1 / 2}\left\|\llbracket \gamma_{h} \cdot \mathbf{n} \rrbracket\right\|_{0, e} .
\end{aligned}
$$

The same argument shows that it holds

$$
\left(g-g_{h}, v_{e}\right) \leq C\left(\sum_{T \subset \omega_{e}} h_{T}^{2}\left(h_{T}^{2}+t^{2}\right)\left\|g-g_{h}\right\|_{0, T}^{2}\right)^{1 / 2} h_{e}^{1 / 2}\left(h_{e}^{2}+t^{2}\right)^{1 / 2}\left\|\llbracket \gamma_{h} \cdot \mathbf{n} \rrbracket\right\|_{0, e} .
$$


We now notice that

$$
\left(\gamma_{h}-\gamma, \nabla v_{e}\right)=h_{e}^{3}\left(\gamma_{h}-\gamma, \nabla \varphi_{e}\right)+h_{e} t^{2}\left(\gamma_{h}-\gamma, \nabla \varphi_{e}\right)
$$

On one hand, using (4.52), we have

$$
\begin{aligned}
h_{e}^{3}\left(\gamma_{h}-\gamma, \nabla \varphi_{e}\right) & \leq\left\|\gamma_{h}-\gamma\right\|_{-1, \omega_{e}} h_{e}^{3}\left|\nabla \varphi_{e}\right|_{1, \omega_{e}} \\
& \leq C\left\|\gamma_{h}-\gamma\right\|_{-1, \omega_{e}} h_{e}^{3 / 2}\left\|\llbracket \gamma_{h} \cdot \mathbf{n} \rrbracket\right\|_{0, e}
\end{aligned}
$$

On the other hand, from (4.51) we get

$$
\begin{aligned}
h_{e} t^{2}\left(\gamma_{h}-\gamma, \nabla \varphi_{e}\right) & \leq t\left\|\gamma_{h}-\gamma\right\|_{0, \omega_{e}} h_{e} t\left\|\nabla \varphi_{e}\right\|_{0, \omega_{e}} \\
& \leq C t\left\|\gamma_{h}-\gamma\right\|_{0, \omega_{e}} h_{e}^{1 / 2} t\left\|\llbracket \gamma_{h} \cdot \mathbf{n} \rrbracket\right\|_{0, e}
\end{aligned}
$$

Therefore, using (4.58) and (4.59) from (4.57) we obtain

$$
\left(\gamma_{h}-\gamma, \nabla v_{e}\right) \leq C\left(\left\|\gamma_{h}-\gamma\right\|_{-1, \omega_{e}}+t\left\|\gamma_{h}-\gamma\right\|_{0, \omega_{e}}\right) h_{e}^{1 / 2}\left(h_{e}^{2}+t^{2}\right)^{1 / 2}\left\|\llbracket \gamma_{h} \cdot \mathbf{n} \rrbracket\right\|_{0, e}
$$

Collecting (4.55), (4.56) and (4.60), we infer from (4.54) that

$$
\begin{aligned}
& h_{e}^{1 / 2}\left(h_{e}^{2}+t^{2}\right)^{1 / 2}\left\|\llbracket \gamma_{h} \cdot \mathbf{n} \rrbracket\right\| \|_{0, e} \leq C\left(\left(\sum_{T \subset \omega_{e}} h_{T}^{2}\left(h_{T}^{2}+t^{2}\right)\left\|\operatorname{div} \gamma_{h}+g_{h}\right\|_{0, T}^{2}\right)^{1 / 2}\right. \\
& \left.\quad+\left\|\gamma_{h}-\gamma\right\|_{-1, \omega_{e}}+t\left\|\gamma_{h}-\gamma\right\|_{0, \omega_{e}}+\left(\sum_{T \subset \omega_{e}} h_{T}^{2}\left(h_{T}^{2}+t^{2}\right)\left\|g-g_{h}\right\|_{0, T}^{2}\right)^{1 / 2}\right) .
\end{aligned}
$$

Hence, from (4.47) we get

$$
\begin{aligned}
h_{e}^{1 / 2}\left(h_{e}^{2}+t^{2}\right)^{1 / 2} \| & \llbracket \gamma_{h} \cdot \mathbf{n} \rrbracket \|_{0, e} \leq C\left(\left\|\gamma_{h}-\gamma\right\|_{-1, \omega_{e}}\right. \\
& \left.+t\left\|\gamma_{h}-\gamma\right\|_{0, \omega_{e}}+\left(\sum_{T \subset \omega_{e}} h_{T}^{2}\left(h_{T}^{2}+t^{2}\right)\left\|g-g_{h}\right\|_{0, T}^{2}\right)^{1 / 2}\right)
\end{aligned}
$$

Estimate (4.28) now follows from (4.30), (4.35), (4.39), (4.47) and (4.62).

Acknowledgments. This work has been supported by the European Project HPRNCT-2002-00284 "New Materials, Adaptive Systems and their Nonlinearities. Modelling, Control and Numerical Simulation". The authors are grateful to G. Sangalli (IMATI-CNR, Pavia) for the useful discussions regarding Lemma 4.1.

\section{REFERENCES}

[1] D.N. Arnold, and R.S. Falk, A uniformly accurate finite element method for the ReissnerMindlin plate, SIAM J. Numer. Anal. 26 (1989), pp. 1276-1290.

[2] F. Auricchio, and C. Lovadina, Partial selective reduced integration schemes and kinematically linked interpolations for plate bending problems, Math. Models Methods Appl. Sci. 9 (1999), pp. 693-722.

[3] F. Auricchio, and C. Lovadina, Analysis of kinematic linked interpolation methods for ReissnerMindlin plate problems, Comput. Methods Appl. Mech. Engrg. 190 (2001), pp. 18-19.

[4] D. Braess, and R. Verfürth A posteriori error estimators for the Raviart-Thomas element, SIAM J. Numer. Anal. 33 (1996), pp. 2431-2444.

[5] F. Brezzi, K.J. Bathe, and M. Fortin, Mixed-interpolated elements for Reissner-Mindlin plates, Internat. J. Numer. Methods Engrg. 28 (1989), pp. 1787-1801.

[6] F. Brezzi, and M. Fortin, Mixed and Hybrid Finite Element Methods, Springer, New York, 1991. 
[7] F. Brezzi, M. Fortin, and R. Stenberg, Error analysis of mixed-interpolated elements for Reissner-Mindlin plates, Math. Models Methods Appl. Sci. 1 (1991), pp. 125-151.

[8] C. Carstensen, Residual-based a posteriori error estimate for a nonconforming ReissnerMindlin plate finite element, SIAM J. Numer. Anal. 39 (2002), pp. 2034-2044.

[9] C. Carstensen, and J. Schöberl, Residual-based a posteriori error estimate for a mixed ReissnerMindlin plate finite element, Preprint.

[10] D. Chapelle, and R. Stenberg, An optimal low-order locking-free finite element method for Reissner-Mindlin plates, Math. Models and Methods in Appl. Sci., 8 (1998), pp. 407-430.

[11] D. Chapelle, and R. Stenberg Stabilized finite element formulations for shells in a bending dominated state, SIAM J. Numer. Anal. 36 (1999), pp. 32-73.

[12] P.G. Ciarlet, The Finite Element Method for Elliptic Problems, North-Holland, 1978.

[13] R. Duran, and E. Liberman, On mixed finite-element methods for the Reissner-Mindlin plate model, Math. Comp. 58 (1992), pp. 561-573.

[14] R.S. Falk, and T. Tu, Locking-free finite elements for the Reissner-Mindlin plate, Math. Comp., 69 (2000), pp. 911-928.

[15] E. Liberman, A posteriori error estimator for a mixed finite element method for ReissnerMindlin plate, Math. Comp. 70 (2000), pp. 1383-1396.

[16] C. Lovadina, A new class of mixed finite element methods for Reissner-Mindlin plates, SIAM J. Numer. Anal. 33 (1996), pp. 2457-2467.

[17] C. Lovadina, Analysis of a mixed finite element method for the Reissner-Mindlin plate problems, Comput. Methods Appl. Mech. Engrg. 163 (1998), pp. 71-85.

[18] M. Lyly, On the connection between some linear triangular Reissner-Mindlin plate bending elements, Numer. Math. 85 (2000), pp. 77-107.

[19] M. Lyly, and R. Stenberg, Stabilized finite element methods for Reissner-Mindlin plates, Forschungsbericht 4, Universität Innsbruck, Institut für Mathematik und Geometrie, (1999).

[20] J. Pitkäranta, Boundary subspaces for the finite element method with Lagrange multipliers, Numer. Math. 33 (1979), pp. 273-289.

[21] R. Stenberg, A new finite element formulation for the plate bending problem, in Asymptotic Methods for Elastic Structures, eds. P.G. Ciarlet, L. Trabucho and J. Viaño, Walter de Gruyter \& Co.,

[22] R.L. Taylor, and F. Auricchio, Linked interpolation for Reissner-Mindlin plate elements: Part II- A simple triangle, Int. J. Numer. Methods Eng. 36 (1993), pp. 3057-3066.

[23] A. Tessler, and T.J.R. Hughes, A three-node Mindlin plate element with improved transverse shear, Comput. Methods Appl. Mech. Engrg. 50 (1985), pp. 71-101.

[24] R. Verfürth, Error estimates for a finite element approximation of the Stokes problem, RAIRO Anal. Numer. 18 (1984), pp. 175-182.

[25] R. Verfürth, A posteriori eror estimation and adaptive mesh-refinement techniques, J. Comput. Appl. Math. 50 (1994), pp. 67-83. 
(continued from the back cover)

A465 Ville Turunen

Function Hopf algebra and pseudodifferential operators on compact Lie groups June 2004

A464 Ville Turunen

Sampling at equiangular grids on the 2-sphere and estimates for Sobolev space interpolation

November 2003

A463 Marko Huhtanen, Jan von Pfaler

The real linear eigenvalue problem in $C^{n}$

November 2003

A462 Ville Turunen

Pseudodifferential calculus on the 2-sphere

October 2003

A461 Tuomas Hytönen

Vector-valued wavelets and the Hardy space $H^{1}\left(R^{n} ; X\right)$

April 2003

A460 Timo Eirola, Jan von Pfaler

Numerical Taylor expansions for invariant manifolds

April 2003

A459 Timo Salin

The quenching problem for the $\mathrm{N}$-dimensional ball

April 2003

A458 Tuomas Hytönen

Translation-invariant Operators on Spaces of Vector-valued Functions

April 2003

A457 Timo Salin

On a Refined Asymptotic Analysis for the Quenching Problem

March 2003 


\section{HELSINKI UNIVERSITY OF TECHNOLOGY INSTITUTE OF MATHEMATICS}

\section{RESEARCH REPORTS}

The list of reports is continued inside. Electronical versions of the reports are

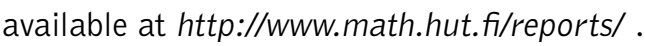

A470 Lasse Leskelä

Stabilization of an overloaded queueing network using measurement-based admission control

March 2004

A469 Jarmo Malinen

A remark on the Hille-Yoshida generator theorem

May 2004

A468 Jarmo Malinen, Olavi Nevanlinna, Zhijian Yuan

On a tauberian condition for bounded linear operators

May 2004

A467 Jarmo Malinen, Olavi Nevanlinna, Ville Turunen, Zhijian Yuan A lower bound for the differences of powers of linear operators May 2004

A466 Timo Salin

Quenching and blowup for reaction diffusion equations

March 2004 